\title{
The Grammar of Politics, through the lens of Surveys and Web-based Social Network methods
}

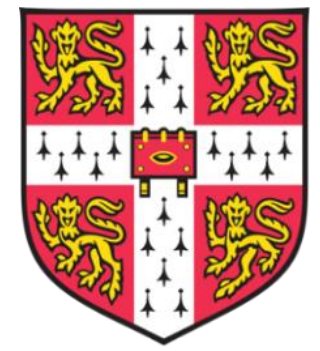

Elizaveta Karmannaya \& Dr Lee de-Wit

Department of Psychology

University of Cambridge

August 2020

Word count: 19,982 


\section{Table of Contents}

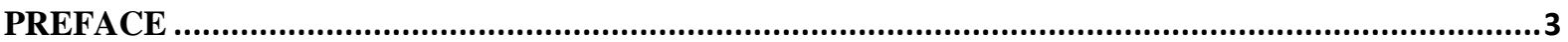

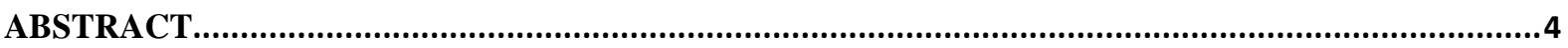

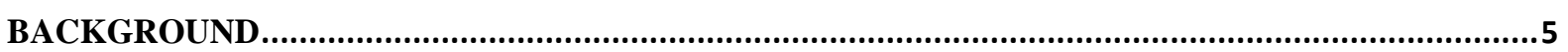

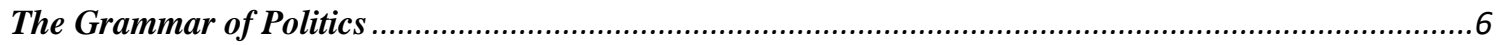

The Mechanisms of Noun use among Social Conservatives .............................................................

Overview of the present research ...................................................................................................

STUDY 1 - A UK-BASED REPLICATION OF CICHOCKA ET AL. (2016), WITH THE ADDITION

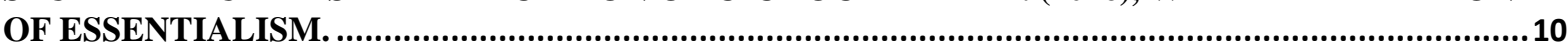

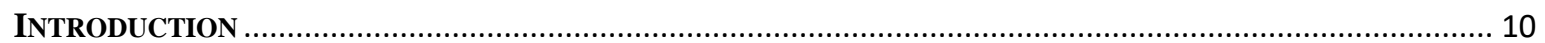

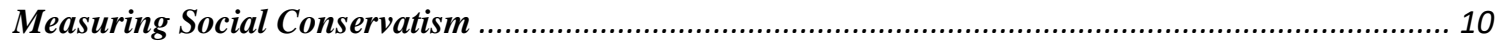

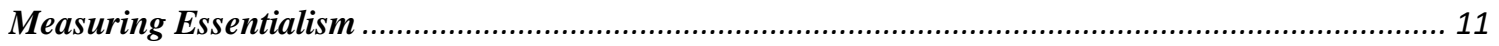

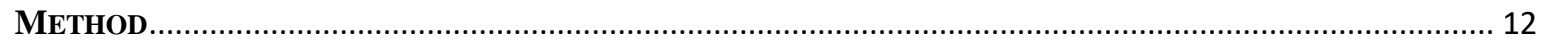

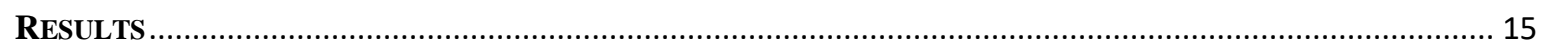

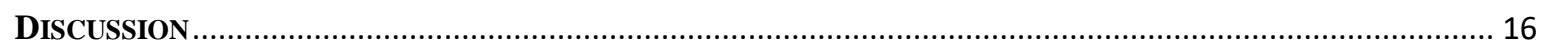

STUDY 2 - NOUN USE AND POLITICAL NETWORKS ON TWITTER...........................................17

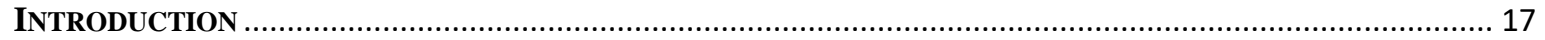

Creating measures of Noun use and Conservatism from Twitter data ............................................. 18

Remaining concerns about Twitter as a research instrument ........................................................... 22

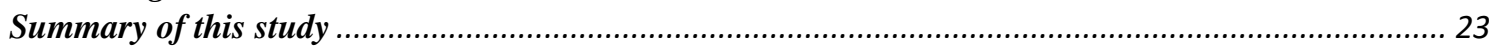

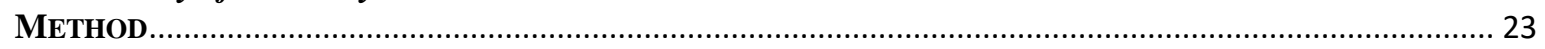

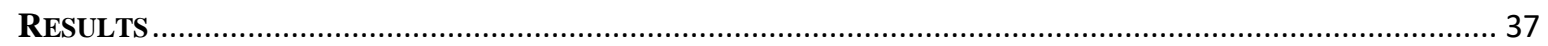

1. Common Nouns, as predicted by Side and Centrality .............................................................. 37

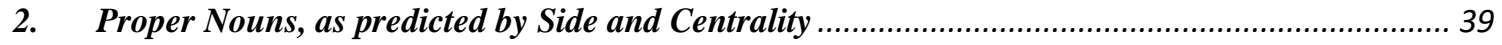

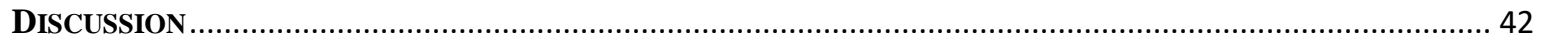

STUDY 3 - COMMON NOUNS, PROPER NOUNS AND SOCIAL CONSERVATISM, WITH ESSENTIALISM AND DECISIVENESS AS POTENTIAL MEDIATORS...........................................46

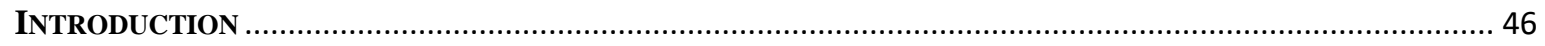

METHOD

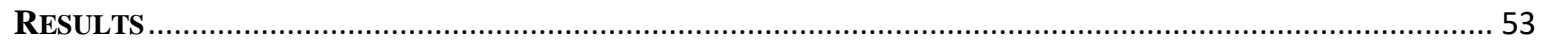

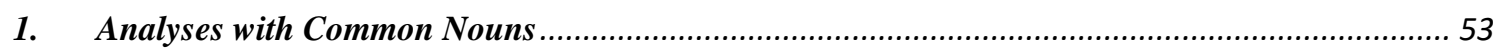

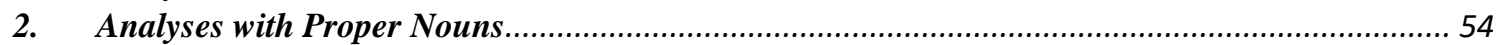

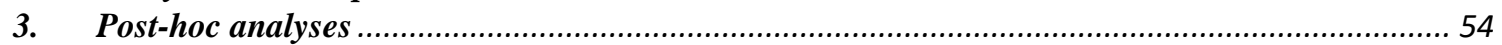

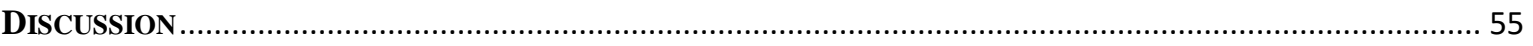

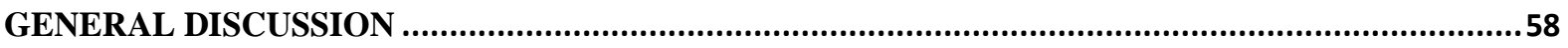

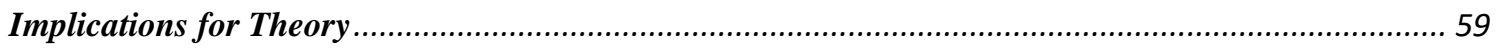

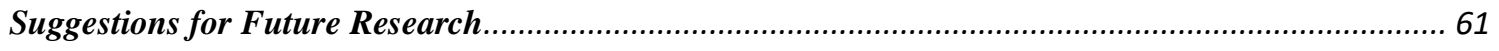

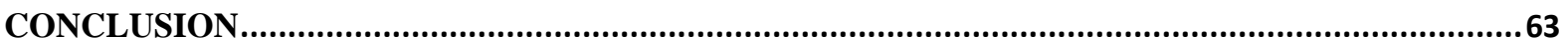

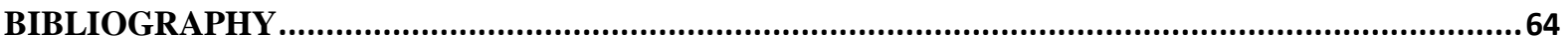




\section{Preface}

\section{Author Contribution:}

Both authors contributed to the design and analysis plan of the study. EK conducted the analysis, collected the data, and wrote the thesis.

\section{Acknowledgements:}

EK would like to express her thanks to the two MPhil examiners, Dr David Stillwell (University of Cambridge) and Dr Jens Koed Madsen (University of Oxford), for their feedback on the thesis, their insightful recommendations, and for the fascinating discussion at the MPhil viva. 


\begin{abstract}
Political conflict and misunderstanding pose dangers to society. Psychological science has the potential to reduce this by providing unique insights into cognitive patterns associated with different political ideologies. Interestingly, seemingly unrelated behaviours have been found to correlate with political attitudes, including grammar use. Cichocka et al. (2016) found that individuals more inclined towards Social Conservatism tended to use more Nouns when completing sentences describing individuals. This three-part study further investigated this association, its manifestations in spontaneous communication, and its underlying mechanisms.

Study 1 replicated the association between Noun use and Social Conservatism in a UKbased survey, using a broader set of political measures. It also found that the trait Essentialism, or the tendency to perceive individuals through stable underlying essences, may mediate the Noun-Conservatism association, alongside differences in education level. Study 2 extended the investigation to spontaneous communication, making use of behavioural and linguistic data from the social networking site Twitter. We built networks of followers of 418 UK political MPs, party leaders and party accounts, and used Network Analysis to infer users' political preferences. Next, we collected $5 \mathrm{mln}$ tweets from a sub-sample of users $(N=33,958)$ and used Natural Language Processing to measure Noun use. Expanding on Cichocka et al.'s (2016) original hypothesis, this study differentiated between Common Nouns and Proper Nouns. It found that Conservatives, on average, used more Proper Nouns per tweet than Liberals, while the effects for Common Nouns were inconsistent across various measures of Noun use. Study 3 aimed to validate the novel finding with Proper Nouns in a controlled questionnaire setting, constructing novel sentence completion items. It did not confirm the association between political attitudes and Proper Noun use, but replicated the original effect with Common Nouns, while also suggesting that it may be underpinned by sex and/or Essentialism.

These findings suggest that the psychological characteristics differentiating Liberals from Conservatives, perhaps including the trait Essentialism, may affect their use of Nouns, although the direction of influence and potential demographic confounds need to be investigated experimentally. These results also suggest that in spontaneous, contextualised communication, such as that on Twitter, these differences may manifest themselves in the use of Proper rather than Common Nouns. Finally, these studies demonstrate the benefits of combining short and simple, yet highly controlled questionnaires with more exploratory and larger-scale, web-based observations of spontaneous language use for the advancement of knowledge in Political Psychology and Psycholinguistics.
\end{abstract}




\section{The Grammar of Politics, through the lens of Surveys and Web-based Social Network methods}

\section{Background}

Political misunderstandings have long been a cause of conflict and societal strain. The field of Political Psychology can potentially help prevent or resolve such misunderstandings by providing a better understanding of the psychological differences between various ideologies. Fascinating individual differences have been found, which correlate with political attitudes: for example, Conservatism, or Right-wing ideology, is associated with lower Big Five trait Openness and higher trait Conscientiousness (Gerber, Huber, Doherty, Dowling, \& Ha, 2010). Conservative and more extreme ideologies are also associated with lower cognitive flexibility (Zmigrod, Rentfrow, \& Robbins, 2018, 2020). Furthermore, Liberals and Conservatives have been shown to base their moral perceptions and decisions on differing sets of Moral Foundations, with Liberals relying more strongly on Care and Fairness, but Conservatives relying on Care and Fairness but also on Authority, Loyalty and Purity (Graham, Haidt, \& Nosek, 2009).

One can argue that these effects are somewhat expected, considering that the items that test these traits often explicitly tap into political attitudes (e.g. "conventional" in Openness, Gosling, Rentfrow, \& Swann, 2003, and "Whether or not someone respected the traditions of society" in Moral foundations, Graham et al., 2009). Yet, seemingly non-political behaviours have also been found to correlate with political tendencies - one example of this is language use (Slatcher, Chung, Pennebaker, \& Stone, 2007; Sylwester \& Purver, 2015; Wojcik, Hovasapian, Graham, Motyl, \& Ditto, 2015). Within the more abstract domain of language form, political differences have been found in the use of grammar, with Conservatives reportedly using more Nouns than Liberals (Cichocka, Bilewicz, Jost, Marrouch, \& Witkowska, 2016). This finding is interesting because it may expose more fundamental, nonideological thought patterns associated with political leaning. In particular, political differences in the use of Nouns may be associated with underlying variation in cognitive needs for certainty (Webster \& Kruglanski, 1994) or differences in the perceptions of individuals' traits as stable and discrete (Bastian \& Haslam, 2006; Carnaghi et al., 2008). These mechanisms are further explored in this research project.

A substantial amount of the reported research is cross-sectional and correlational, so the direction of potential influence remains largely unknown. Nevertheless, a growing body of work suggests that knowledge of these psychological differences may improve communication 
across the political spectrum. For example, evidence from 'moral reframing' work shows that framing a position traditionally unsupported by certain political groups (e.g. environmental protection policy in American Conservatives) to align more closely with their moral values (e.g. in terms of Purity more so than Care) may lead to more effective persuasion (Feinberg \& Willer, 2012; see Feinberg \& Willer, 2019, for a review). Similar 'reframing' may also be possible in the linguistic domain: a recent intervention study demonstrated that using certain grammatical constructions over others in describing intergroup conflict can reduce anger and improve policy support in the Israeli-Palestinian conflict (Idan, Halperin, Hameiri, \& Reifen Tagar, 2018). These findings demonstrate the practical value of this field of research.

\section{The Grammar of Politics}

A questionnaire study from Cichocka et al. (2016) recently found that more Socially Conservative individuals chose to use more Nouns than Liberals when completing sentences describing people (e.g. the equivalents of "Anna saved a friend from drowning. Anna ... is a hero [Noun]/acted heroically [Verb/Adjective]"). Cichocka et al.'s findings came from two samples - 189 participants from Poland and 100 from Lebanon, differing in both political and linguistic context. In the Polish sample, Cichocka et al. differentiated between Implicit (i.e. policy preferences) an Explicit measures of Social Conservatism (i.e. self-placement on a political scale). A difference in Noun use was found in both measures and in both national political contexts. Furthermore, going beyond single-sentence questionnaire measures, Cichocka et al.'s third study also confirmed the effect in an analysis of 101 American presidential speeches from 13 presidents.

However, the finer details of Cichocka et al.'s (2016) findings remain unclear. A replication attempt using a questionnaire adapted to the USA (Crawford, 2018) confirmed the Noun-Conservatism association, but only in the Explicit measure of political orientation, and not in Implicit policy preferences. Additionally, no study so far has tested this effect in realworld spontaneous communication, outside of questionnaires or formal speeches.

Furthermore, key questions about the effect of Conservatism on Noun use remain unanswered. The first is about the direction of potential influence-does a preference for Nouns lead one to develop more Socially Conservative attitudes, relating to the popular Sapir-Whorf hypothesis (e.g. Koerner, 1992), or vice versa? This is difficult to answer as language or politics cannot be easily manipulated in a causal, experimental design. Another question arises over the underlying mechanisms - could certain psychological traits mediate the relationship? This 
is possible to investigate using questionnaire data, and formed one of the goals of the present research.

\section{The Mechanisms of Noun use among Social Conservatives}

Why might Social Conservatives prefer Nouns? Nouns, rather than Verbs or Adjectives, are believed to provide more abstract descriptions of individuals (Fiedler, Semin, \& Bolten, 1989) or situations (Idan et al., 2018). Based on this, Cichocka et al. (2016) suggested that the association of Nouns with Social Conservatism may be related to the cognitive trait Need for Closure - a latent behavioural trait which corresponds to "a desire for a firm answer to a question and an aversion toward ambiguity" (Webster \& Kruglanski, p.264). Greater Need for Closure is associated with both political Conservatism (Jost, Glaser, Kruglanski, \& Sulloway, 2003) and greater linguistic intergroup bias (Webster, Kruglanski, \& Pattison, 1997), i.e. the increased use of linguistic abstraction when describing positive behaviours of in-group members or negative behaviours of out-group members (Maass, 1999).

Two distinct facets of Need for Closure have been identified - Personal Need for Structure and Decisiveness (Mannetti, Pierro, Kruglanski, Taris, \& Bezinovic, 2002; Neuberg, Judice, \& West, 1997). Personal Need for Structure refers specifically to the preference for predictability, order and structure, and a discomfort with ambiguity (Neuberg et al., 1997; Webster \& Kruglanski, 1994), and was found to statistically mediate the Noun-Conservatism association in Cichocka et al. (2016). Yet, this was only tested in the first of Cichocka et al.'s three studies, and Personal Need for Structure was not associated with Noun use in Crawford's (2018) USA-based replication. Decisiveness - the other facet of Need for Closure, which measures preferences for quick, decisive answers (Neuberg et al., 1997; Webster \& Kruglanski, 1994) - was unrelated to a preference for Nouns in Cichocka et al., but correlated with both Noun use and Explicit Conservatism in Crawford's replication, thus providing grounds for a potential mediation analysis (not performed by Crawford).

Thus, the influence of the two facets of Need for Closure on Noun use and Social Conservatism has not been consistently demonstrated in past research. The present set of studies propose an alternative explanation. Semin and Fiedler (1991) suggested that Nouns facilitate more discrete, categorical perceptions and perceptions of internal causality and stability. Such perceptions define the trait Essentialism, or one's tendency to perceive and categorise individuals through their underlying unchanging essences (Haslam, Rothschild, \& Ernst, 2000). Explicit associations between Nominal constructions and essentialist thinking 
have been demonstrated experimentally (Carnaghi et al., 2008; Graf, Bilewicz, Finell, \& Geschke, 2013; Walton \& Banaji, 2004). For example, Carnaghi et al. (2008) presented a large group of participants with personal descriptions containing Adjectives (e.g. "Mark is Catholic") or Nouns (e.g. "Mark is a Catholic"), and asked them to estimate the frequency of descriptor-congruent behaviours performed by the described individuals (e.g. "How often does he attend church in a year?"). They found that more essentialist inferences, i.e. those involving a greater frequency of the descriptor-congruent behaviour, were made following the descriptions containing Nouns. Carnaghi et al. (2008) also demonstrated that Nouns inhibited alternative classifications of individuals (e.g. participants who read about an individual being described as "an athlete" rather than "athletic" were less likely to ascribe other characteristics, e.g. "artist/artistic", to the same individual). Most interestingly, Carnaghi et al. showed that the relationship holds in both directions: participants who read stories describing successful sportsmen or intelligent individuals in terms of genetic predispositions rather than training were more likely to describe them using Nouns ("an athlete" or "a genius") than Adjectives ("athletic" or "brilliant"), with the effect remaining consistent across two languages (Italian and German) and various group memberships (athletics, religion etc.).

Interestingly, this stabilising and categorising effect of Nouns is not only evident in adults (Walton \& Banaji, 2004), but also in children as young as five (Gelman \& Heyman, 1999), and not only in inferences about others (e.g. Carnaghi et al., 2008; Gelman \& Heyman, 1999), but also inferences about oneself (Walton \& Banaji, 2004). Thus, Essentialism is a trait strongly facilitated by the use of Nouns, with a potential bi-directional influence.

In the political domain, Essentialism has been used to explain the finding that nominal descriptions of individuals' nationality enhance in-group national favouritism (Graf et al., 2013). At the same time, nationalistic attitudes, which are common in Social Conservatives, are associated with the tendency to process even non-political information in more categorical ways in tasks such as the Wisconsin Card Sorting Test (Zmigrod et al., 2018). Tying these together, higher Essentialism directly predicts anti-Immigration attitudes in Australia (Bastian \& Haslam, 2008). Finally, a potential precursor of psychological Essentialism, the Inherence Heuristic (Cimpian \& Salomon, 2014), predicts endorsement of Conservative views (e.g. that socioeconomic stratification is acceptable, Hussak \& Cimpian, 2018) and the tendency towards nominal realism - the belief that a name is rooted in the inherent features of its properties (Sutherland \& Cimpian, 2015).

Applying this to the present research questions, a mechanism is possible whereby Social Conservatives, due to their stronger trait Essentialism, become more likely to perceive 
individuals such as foreign nationals as unchanging or strongly influenced by their underlying essences. The Conservative individual might therefore prefer to describe these foreign nationals using Nouns, and might also develop anti-Immigration attitudes (for example, if they fear that immigrants may not assimilate and damage their country's culture). The present research explored this mechanism using mediation analyses, but does not claim any causality.

\section{Overview of the present research}

The following collection of studies aimed to provide a deeper understanding of the Noun-Conservatism association, its external validity beyond responses in a questionnaire, and its potential underlying mechanisms. Study 1 aimed to replicate the Noun-Conservatism association in a UK sample, using the same Noun questionnaire items as Crawford (2018), and also explored a novel hypothesis that the trait Essentialism may act as a potential mediating variable. Study 2 extended the original finding to spontaneous, real-world communication by collecting over 5 million tweets from over 33,000 users from the social networking site Twitter. Study 2 applied network analysis to all Twitter 'followers' of British Members of Parliament (MPs) to create measures of political leaning, and used a pre-trained Twitter-specific part-ofspeech tagger (Owoputi et al., 2013) to count Nouns in their tweets. Expanding on the original hypothesis, Study 2 also distinguished between Proper Nouns and Common Nouns. Finally, Study 3 attempted to validate the results from Twitter in a more controlled questionnaire, using a separate, larger UK sample, and constructing novel Proper Noun sentence completion items in a format similar to the original stimuli from Cichocka et al. (2016). Study 3 also re-tested the original association of Conservatism with Common Nouns, on both Implicit and Explicit measures, and tested for the potential mediating roles of both Essentialism and Decisiveness. 


\section{Study 1 - A UK-based Replication of Cichocka et al. (2016), with the addition of Essentialism.}

\section{Introduction}

This study pursued two goals: a UK-based replication of Cichocka et al. (2016) and an exploration of the influence of trait Essentialism on the Noun-Conservatism association. The discussion below outlines methodological decisions made in relation to these goals.

\section{Measuring Social Conservatism}

As described, Cichocka et al. (2016) used a questionnaire, recruited two samples from Poland and Lebanon and found that Social Conservatism correlates with the tendency to use Nouns rather than Verbs or Adjectives in sentence completion. Cichocka et al. found this effect on both Implicit and Explicit measures of Social Conservatism in their first study. However, the association with Implicit policy preferences was not tested in the Lebanese sample in their second study, and was not replicated by Crawford (2018) in the USA, so the effects of Implicit Social Conservatism in particular remain uncertain.

Additionally, no follow-up study has yet been done using a more general measure of Implicit Social Conservatism, going beyond specific policy attitudes. The relative consistency of Cichocka et al.'s (2016) results across Poland, Lebanon and the USA suggests that the association between Social Conservatism and Noun use may be a universal phenomenon. At the same time, however, Social Conservative attitudes differ widely across countries, with Conservatives in the UK and USA, for example, generally being against Immigration, but Conservatives in Canada generally supporting pro-Immigration and pro-refugee policies. Thus, any general measure of Social Conservatism is still unlikely to apply across the globe.

To tap into the underlying traits and beliefs of British Social Conservatives, two measures are frequently used: Right-Wing Authoritarianism (Altemeyer, 1981) and antiImmigration attitudes, with Social Conservatives usually scoring higher on both (Fieldhouse et al., 2018). Right-Wing Authoritarianism, or RWA (Altemeyer, 1981), is an ideological variable which describes one's willingness to submit to established authorities, to adhere to societal norms and to remain hostile towards people who do not adhere to the same norms (example item: "Schools should teach children to obey authority."). Despite discussions of this trait's multi-faceted structure (Duckitt, Bizumic, Krauss, \& Heled, 2010; Mavor, Louis, \& Sibley, 2010), RWA questionnaires as short as five items have been used in national surveys to assess Social Conservatism in the UK (e.g. Bale, Cheung, Cowley, Wager, \& Menon, 2020; Fieldhouse et al., 2018) and in other countries (e.g. Harnish et al., 2018). Of note, 
Authoritarianism is not uniquely Right-wing (Eysenck, 1981) and can be demonstrated in Leftwing individuals using a similar scale but targeted towards acceptance of Liberal authoritarian leaders (Conway III, Houck, Gornick, \& Repke, 2018). Furthermore, RWA does not cover the anti-Immigration aspect of UK Social Conservative policies (e.g. Terrizzi, Shook, \& McDaniel, 2013). Thus, RWA on its own may not provide a strong enough signal of Social Conservatism for this study, and Immigration-specific items should also be included.

Anti-Immigration attitudes can be assessed with a questionnaire as short as four items, as in the British Election Study (Fieldhouse et al., 2018). This study used Cronbach's alpha to ensure that RWA and Immigration are actually measuring a consistent underlying construct (i.e. that of Social Conservatism), and then combined the two to create a more general measure of Implicit Social Conservatism. This study did not consider Economic Conservatism due to a lack of theoretical foundation for this association, and because no such association was discovered in the original study (Cichocka et al., 2016).

\section{Measuring Essentialism}

The second goal of this study was to explore the influence of Essentialism on Conservatives' preference for Nouns. Essentialism is a multi-faceted variable, and its measures have been divided into those investigating beliefs in biological (Bastian \& Haslam, 2006) or social determinism (Rangel \& Keller, 2011), discreteness, informativeness (Bastian \& Haslam, 2006), or the stability of individuals' basic characteristics (Levy, Plaks, Hong, Chiu, \& Dweck, 2001). Higher scores on a combination of these scales correlate with anti-Immigration attitudes in Australia (Bastian \& Haslam, 2008). Because Noun use and Social Conservatism may be associated with categorical thinking about people and with perceptions of internal stability (Bastian \& Haslam, 2008; Carnaghi et al., 2008), this study predicted that two aspects would be most relevant to the Noun-Conservatism association: Discreteness, or the extent to which someone believes that individuals can be organised into discrete categories (example item: "Everyone is either a certain type of person or they are not.", Bastian \& Haslam, 2006), and Implicit Person Theory, or the extent to which one believes that people's attributes are fixed and unmalleable (example item: "People can do things differently but the important parts of who they are can't really be changed"; Levy et al., 2001). This study investigated these two Essentialism measures as potential mediators of the Noun-Conservatism association. 


\section{Method}

Following the guidelines by Simmons, Nelson and Simonsohn (2012), throughout this entire paper we report how we determined our sample size, all data exclusions, all manipulations, and all measures used in the studies. Relevant data, code and materials for all three studies are available on the Open Science Framework (https://osf.io/dr7bk/?view_only=27f913c49c7b48019484f784b5db4135).

\section{Participants}

A sample of 347 participants was recruited through Prolific Academic, and paid $£ 4.10$ for an estimated 30 minutes of their time. This sample size was determined by a power analysis based on the smallest of Cichocka et al.'s (2016) effect sizes ( $r=.15)$, and using the conventional alpha $=.05$ and beta $=.80$ levels. Participants were recruited in waves of between 20 and 50, and in every wave the balance of political leanings was checked. To achieve a similar amount of Liberal and Conservative participants overall, in one of these waves we explicitly requested 43 participants who voted 'Conservative' in the 2017 General Election (see Appendix A for details). All recruitment took place between $18^{\text {th }}$ and $31^{\text {st } J u l y ~ o f ~ 2019 . ~ I n ~}$ this study, we supplied the reported questionnaire items, but the data was collected by two Laidlaw Scholars at University College London.

Twenty-one participants were excluded for incorrectly answering the two attention check questions, as well as two individuals who were under 18 years old. This resulted in a sample of 324 participants - 170 female and 153 male (one preferred not to say), aged between 18 and $72(M=39.31, S D=14.69)$.

\section{Procedure}

Participants filled out a questionnaire hosted on Qualtrics Survey Software. This consisted of three groups of sub-questionnaires - Noun use, Social Conservatism and Essentialism (five sub-questionnaires in total, presented in a randomised order). Demographics (gender, age and education level) were collected at the end. The questionnaire was part of a larger 30-minute survey and also included questions and stories as measures of attributional bias, and scales measuring other facets of Essentialism and economic political attitudes. These were not related to the present investigation and thus are not included in this analysis. 


\section{Materials}

Noun score:

This study re-used Crawford's (2018) 10 Noun items in English (e.g. "Peter was chewing loudly during family dinner. Peter ... is an annoyancelacted annoyingly."). The order of the items and the response options were not randomised - five of them had the Noun option first, and five the Adjective/Verb option first (see Appendix B). As in the original study (Cichocka et al., 2016), Noun answers were scored as 1, and Adjective/Verb answers as 0, and the final score was a sum of the 10 items. Descriptive statistics are reported in Table 1 below.

\section{Implicit Social Conservatism:}

To measure Implicit Social Conservatism, short versions of both the RWA (five items) and Immigration questionnaires (four items) were adapted from the British Election Study (BES) 2014-2018, waves 1 to 13 (Fieldhouse et al., 2018). The RWA scale consisted of five items (e.g. "Schools should teach children to obey authority."), and participants responded on a 5-point Likert scale (from 1=strongly disagree to 5=strongly agree). The resulting score was an average of the five items, with higher scores indicating greater Authoritarianism.

The Immigration scale from the BES consisted of four items (e.g. "Do you think immigration is good or bad for Britain's economy? Please choose a rating. ") and participants responded on Likert scales varying in length from five to seven items (e.g. 1=Bad for economy to 7=Good for Economy). Minor changes were made to the BES items: one question, which originally asked participants to place themselves and the UK political parties on a scale of 1 to 10 , was changed to only ask about participants themselves, and was scored on a shorter, sevenpoint scale in order to keep this survey short and consistent. Furthermore, this study removed the 'Don't know' option in all of the Immigration items, and reverse-scored all four items for ease of interpretation. The resulting Immigration score is an average of the $z$-scores of the four items, with higher scores indicating an aversion to Immigration.

The RWA and Immigration items are attached in Appendix B. Descriptive statistics for these two scores are reported in Table 1. Aiming to combine these two scales into one Implicit Social Conservatism score, we z-scored each scale and checked their internal consistency. This turned out to be high (Cronbach's alpha=0.88), suggesting that the two scales indeed measured a consistent underlying construct. Thus, the $z$-scores of these two scales were averaged to represent Implicit Social Conservatism, with higher values indicating greater Conservatism. 


\section{Essentialism:}

As discussed, Discreteness (Bastian \& Haslam, 2006) and Implicit Person Theory (IPT, Levy et al., 2001) were chosen as measures of Essentialism. The Discreteness scale consisted of eight items (e.g. "People can behave in ways that seem ambiguous but the central aspects of their character are clear cut. "; Bastian \& Haslam, 2006), and participants responded on a five-point Likert scale (from 1=strongly disagree to 5=strongly agree). Four of the items were reverse scored (see Appendix $B$ ). The final score was an average of the eight items, with higher scores indicating greater Essentialism.

The IPT scale consisted of three items (e.g. "The kind of person someone is, is something basic about them and it can't be changed very much."; Levy et al., 2001), with answer options again on the same five-point Likert scale. The final score was an average of the three items, also with higher scores indicating greater Essentialism.

A combination of IPT and Discreteness scores did not show good internal consistency (Cronbach's alpha=0.50), so these scales were kept separate. Descriptive statistics are presented in Table 1. To correct for the inclusion of two different measures of Essentialism, Holm-Bonferroni correction was used. Only the corrected $p$-values are reported below.

\begin{tabular}{|c|c|c|c|c|c|c|c|}
\hline Measure & Items & $M$ & $S D$ & Median & Range & Skew & Kurtosis \\
\hline Noun score & 10 sentences & 3.13 & 1.85 & 3 & $0-8$ & 0.15 & -0.63 \\
\hline $\begin{array}{l}\text { Implicit } \\
\text { Social }\end{array}$ & $R W A-5$ Likert scales & 2.61 & 0.87 & 2.60 & $1-5$ & 0.42 & -0.24 \\
\hline Conservatism & $\begin{array}{l}\text { Immigration-4 Likert } \\
\text { scales, reverse-scored } \\
\text { and } z \text {-scored }\end{array}$ & 0 & 0.89 & -0.04 & $\begin{array}{c}-1.85- \\
1.76\end{array}$ & 0.18 & -0.61 \\
\hline $\begin{array}{l}\text { Discreteness } \\
\text { (Essentialism) }\end{array}$ & 8 Likert scales & 2.82 & 0.60 & 2.88 & 3.25 & -0.05 & -0.40 \\
\hline $\begin{array}{c}I P T \\
\text { (Essentialism) }\end{array}$ & 3 Likert scales & 3.07 & 0.87 & 3 & $1-5$ & -0.16 & -0.91 \\
\hline
\end{tabular}

Table 1. Descriptive statistics for the measures used.

\section{Design}

This study followed a correlational design, with Noun scores as the outcome variable, Social Conservatism as the key predictor, and Discreteness and IPT as additional predictors and potential mediators of the relationship. 


\section{Results}

Pairwise correlations were computed with the aim of replicating Cichocka et al.'s (2016) and Crawford's (2018) results. This study too found that higher Noun scores were associated with stronger Implicit Social Conservatism ( $r=0.154, p=.005)$, with a similar effect size to Cichocka et al. (2016). Noun use also correlated positively with two measures of Essentialism: Discreteness $(r=0.182, p=.002)$ and IPT $(r=0.141, p=.011)$. Finally, Implicit Social Conservatism was also associated with both Discreteness $(r=0.311, p=1.11 \mathrm{e}-08)$ and IPT $(r=0.234, p=2.05 \mathrm{e}-05)$. These two Essentialism scales were moderately correlated with each other $(r=0.426, p=1.09 \mathrm{e}-15)$. The distributions of each variable and pairwise correlation plots are presented in Appendix $C$.

Next, this study investigated the role of Essentialism in the relationship between Noun use and Social Conservatism. Mediation analyses were performed in the $R$ package lavaan, predicting Noun use first from Implicit Social Conservatism only (reported above), and then adding Discreteness (Model 1) or IPT (Model 2) as potential mediators.

Model 1 was significant overall $(b=.387, S E=0.138, p=.005)$. As summarised in Figure 1 below, the regression of Social Conservatism on Discreteness was positive and statistically significant $(b=0.265, S E=0.045, p<.001)$, as was the regression of Discreteness on Noun use $(b=0.437, S E=0.169, p=.010)$. Once Discreteness was included, the effect of Social Conservatism on Noun use was no longer significant $(b=0.271, S E=0.144, p=.059)$. This suggests that Discreteness may fully mediate the Noun-Conservatism association.

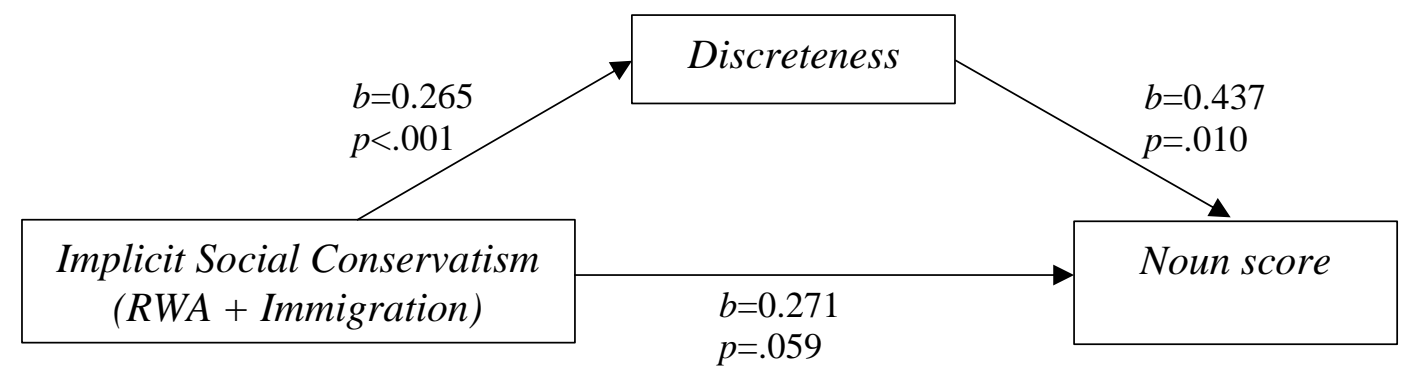

Figure 1. Mediation analysis results for Model 1.

Model 2 was also significant overall ( $b=.387, S E=0.138, p=.005)$. As summarised in Figure 2, the regression of Social Conservatism on IPT was positive and statistically significant ( $b=0.273, S E=0.063, p<.001)$, but the regression of IPT on Noun score was only marginally significant $(b=0.239, S E=0.121, p=.048)$. The effect of Social Conservatism on Noun use remained positive and significant after the inclusion of IPT $(b=0.321, S E=0.141, p=.023)$, suggesting that IPT did not fully mediate the Noun-Conservatism association. 


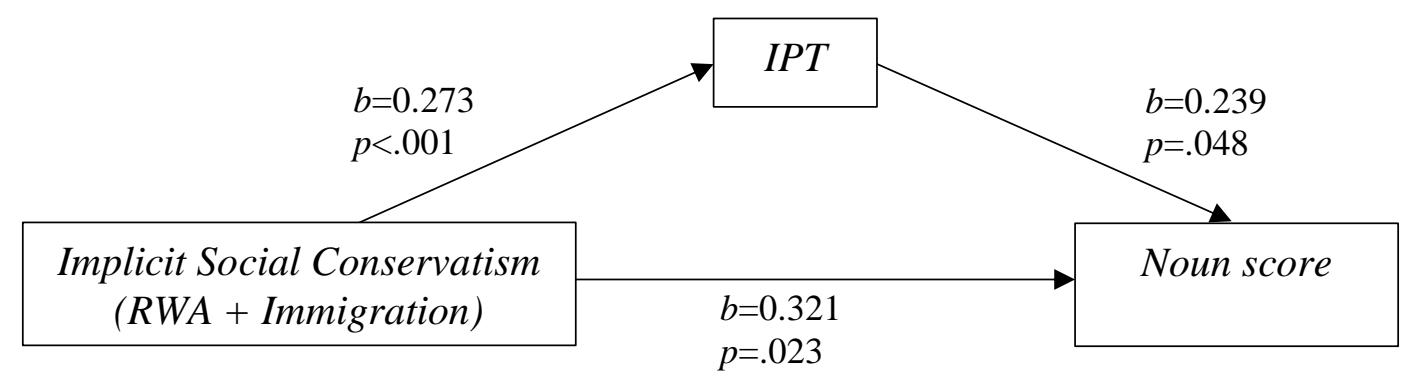

Figure 2. Mediation analysis results for Model 2.

Finally, the influence of demographic variables on Noun use was also analysed. There were no associations between Noun scores and gender $(p=.528)$ or age $(p=.928)$. Education, on the other hand, was negatively correlated with Noun scores $(p=.011, r=-0.141)$, and negatively correlated with Social Conservatism ( $p=2.006 \mathrm{e}-07, r=-0.284)$. Education was also negatively correlated with IPT ( $p=.012$, $r=-0.139)$, and marginally negatively correlated with Discreteness $(p=.047, r=-0.110)$. A regression model predicting Noun use from Social Conservatism, Discreteness, IPT and education was overall significant ( $p=8 \mathrm{e}-04, R^{2}=4.6 \%$ ), but only Discreteness came out as a significant predictor of Noun use $(b=0.372 p=.042)$.

\section{Discussion}

This study pursued two goals: a UK-based replication of the Noun-Conservatism association, originally reported by Cichocka et al. (2016), and a test for potential mediating effects of the trait Essentialism. Meeting the first goal, this study found that greater Implicit Social Conservatism, measured using the more general scales RWA and anti-Immigration, indeed predicted greater Noun scores in a sample of UK-born native English speakers. This replication strengthens the evidence for greater Noun use in Conservatives beyond Poland, Lebanon and the US (Cichocka et al., 2016; Crawford, 2018).

This study also found that Essentialism, as measured by the Discreteness and IPT scales, is associated with both greater Noun use and higher Social Conservatism. The results of the mediation analysis suggested that the political difference in grammar use may be rooted in the tendency to categorise individuals into discrete groups or types, reflected specifically in the trait Discreteness. Furthermore, a regression analysis controlling for education level - a demographic variable not explored in past research in this field (Cichocka et al., 2016; Crawford, 2018), suggested that Discreteness plays a stronger role than politics or demographics in predicting Noun use. Nevertheless, the sample and effect sizes in this study were not large enough to claim full mediation, so further investigation into the facets of Essentialism is necessary. 


\section{Study 2 - Noun use and Political Networks on Twitter.}

\section{Introduction}

This study aimed to build on existing research by investigating the original NounConservatism association in spontaneous speech, collecting data from the social networking site Twitter.

Twitter is a popular site, used by over $20 \%$ of Britain's population (over $15 \mathrm{mln}$ UK users; Statista, 2020). The site provides highly relevant linguistic and behavioural data for the present research question - users on Twitter create personal accounts, 'follow' other users (who can be both everyday users and verified 'elite' accounts, such as politicians, celebrities etc.), and produce 'tweets' of up to 280 characters in length. Additionally, Twitter users can 'mention' each other in tweets (using the “@ ”symbol), and 'like' or 're-tweet' other messages, providing great potential for a scientific assessment of multi-dimensional social interaction. Twitter data can be quickly collected at little or no monetary cost through the Twitter Application Programming Interface (API).

Twitter could significantly improve our scientific understanding of the association between Noun use and political Conservatism for two key reasons. Firstly, Twitter provides data concerning vast amounts of social network interactions between millions of British citizens and hundreds of political leaders, as well as linguistic data within tweets in much larger quantities than the ten single-sentence items used in Study 1. Secondly, unlike questionnaire studies, Twitter contains samples of spontaneous language use. Similar to Cichocka et al.'s (2016) Study 3, Twitter can improve the external validity of this research by providing data on Noun use in real-world communication. It provides an additional strength over the analysis of political speeches (as used by Cichocka et al.). Political speeches are formally written and thus potentially demonstrate strategic goals rather than sub-conscious psychological influences; Twitter, on the other hand, provides short, spontaneous, self-generated speech samples more likely to act as a window into the underlying ideology or psychology of large populations of voters.

However, alongside these advantages, Twitter data is also subject to numerous biases (e.g. Olteanu, Castillo, Diaz, \& Kiciman, 2019), is confounded by automated 'bot' accounts, and raises ethical concerns. These issues are addressed below in relation to the present research question and the corresponding measures employed in this study. 


\section{Creating measures of Noun use and Conservatism from Twitter data}

To extend the exploration from Cichocka et al. (2016) and Study 1 to Twitter, two measures needed to be created: Noun use and political leaning. Similar to Cichocka et al.'s Study 3, the dependent variable Noun use was created by counting Nouns in natural speech. First, a part-of-speech tagging model (Owoputi et al., 2013) was applied to a corpus of tweets. Then, the number of Noun tags was calculated as a percentage of all part-of-speech tokens in each tweet. To account for the presence of Twitter-specific tokens such as hashtags or “@”mentions, which do not constitute a part of the grammatical sentence, and for potentially inconsistent uses of these and other tags by various users, three percentage scores were created.

Importantly, the part-of-speech tagger used distinguished Common Nouns from Proper Nouns. Common Nouns (e.g. "country”) are used to refer to general categories of entities, or 'universals' (Hobbes, 1968), whereas Proper Nouns (e.g. "England”) refer to a specific entity within a category (Bredart, Brennen, \& Valentine, 2002). Little is known about the associations between the use of Proper Nouns and political leanings. Since Proper Nouns are a type of Noun, this study predicted that Conservatives would also use Proper Nouns more than Liberals. However, more generally, there is evidence from lesion studies (Swanson et al., 2020), aphasic patients (Robson, Marshall, Pring, Montagu, \& Chiat, 2004) and developmental literature (Hall, 1991) that Proper and Common Nouns are learnt and processed differently in the brain, with Proper Nouns demanding more cognitive effort to memorise. Furthermore, Common Nouns and Proper Nouns may affect cognition differently - Common Nouns create constructs that are perceived as abstract (Walton \& Banaji, 2004), and Cichocka et al. (2016) argued that this abstraction may underlie the Noun preferences in Conservatives. On the other hand, Proper Nouns, by definition, describe specific entities in the world, thus lessening this abstraction. Therefore, it remains unclear whether Common and Proper Nouns would both be associated with political Conservatism. Thus, these were kept separate throughout this study.

To create the main predictor variable, Conservatism, this study relied on the principle of homophily applied to Twitter. Homophily, or assortative mixing, is the tendency of individuals to form ties with other individuals with similar opinions or behaviours (McPherson, Smith-Lovin, \& Cook, 2001). Other methods of inferring political leaning exist, including language-based methods (Conover, Goncalves, Ratkiewicz, Flammini, \& Menczer, 2011; Preoţiuc-Pietro, Liu, Hopkins, \& Ungar, 2017) or unsupervised network clustering methods (Himelboim, McCreery, \& Smith, 2013). However, as Language was the outcome variable in this investigation, behavioural data was used instead to infer ideology, and since no matched 
data was available to validate unsupervised models, focus was placed on the homophily assumption.

On Twitter, users interact in networks, manifesting homophily by 'following', 'mentioning', 're-tweeting' or 'liking' content from similar users, including those with similar political leanings (Barberá, 2015; Conover, Gonçalves, Flammini, \& Menczer, 2012; Halberstam \& Knight, 2016; Šćepanović, Mishkovski, Gonçalves, Nguyen, \& Hui, 2017; Wu, Hofman, Mason, \& Watts, 2011). Interactions on Twitter are often considered as two-mode one occurring between regular users who may be friends or acquaintances, and the other occurring between major public figures, organisations or celebrities, and their audiences (Boutyline \& Willer, 2017). The sample of interest in this exploration was regular users, or 'voters'. The interactions of these voters with political public figures or parties, collectively titled 'elites', can be used to infer the voters' political leanings (Barberá, 2015; Conover, Ratkiewicz, et al., 2011; Gu, Chen, Sun, \& Wang, 2016; Zamal, Liu, \& Ruths, 2012). Thus, this study constructed networks of users who interact with key UK political elites - MPs, political party accounts or Members of European Parliament (MEPs). A large number of British MPs have official Twitter accounts (up to 579 out of 650; Agarwal, Sastry, \& Wood, 2019), and many of them are actively involved in Twitter communication (Agarwal et al., 2019; Jackson \& Lilleker, 2011).

Of the four types of Twitter interactions (following, mentions, re-tweets and likes), this study chose following patterns as the signal of interest. This was done to minimise the influences of activity bias and sampling bias while maximising ease of interpretation, as discussed subsequently.

Activity bias on Twitter means that not all users contribute equally. For example, in the US, $80 \%$ of all tweets, and $97 \%$ of all tweets about politics come from only the most active 10\% of Twitter users (Pew Research Center, 2019a). Thus, using within-tweet interactions such as mentions or re-tweets to construct the network would only capture those users who actively engage in these behaviours. A similar bias may exist in Twitter likes too. Following patterns, on the other hand, are likely to be more representative of the whole Twitter sample - all users, even the less active ones, follow some other users.

Networks constructed from re-tweets or mentions are also subject to sampling bias (González-Bailón, Wang, Rivero, Borge-Holthoefer, \& Moreno, 2014). Sampling bias is the measurement error introduced by the design of the Twitter API, arising because of the API's rate limits on the number of tweets that can be searched (up to 180 queries per 15 minutes) or collected in real-time from the Streaming API (capped at $1 \%$ of the complete Twitter stream). 
Following networks are likely to be more representative because they avoid this sampling bias, allowing researchers to collect all followers of a user (e.g. an elite) before sampling their tweets.

Another key advantage of following networks is that their interpretation is less prone to error than other interactions. Re-tweets cannot simply be interpreted under homophily as posts may be re-tweeted in disagreement with the original author (this is why many Twitter users state "retweets do not signal endorsement" in their profile descriptions). A similar concern exists with mentions - oftentimes, a tweet disagrees or directly confronts the mentioned user. Thus, without a detailed qualitative analysis of the tweets themselves, retweets or mentions cannot be treated as signals of ideological agreement. The final signal that could have been considered is Twitter likes, created by the click of a 'like' button underneath a tweet. Theoretically, these would most likely signal endorsement of the post. Unfortunately, data on likes is not currently available from the Twitter API.

Thus, based on the homophily assumption, this study treated the choice of a user to 'follow' a political elite as a signal of the user's ideological position being similar to that of the followed elite (for a similar approach, see Barberá, 2015). One potential criticism of this approach is that some users may follow elites that they disagree with too, in cases where these elites are their local representative, the user is interested in learning opposing opinions, or as a 'hate-follow'. However, it is likely that a user who follows elites with opposing views may also follow elites whose opinions they support. This study groups such users under the 'centrist' category and later excludes them, as described below, to allow us to rely on the homophily assumption. Furthermore, homophily is more likely to hold true nowadays due to the phenomenon of algorithmic confounding - since 2010, network ties on Twitter are influenced by the platform's algorithmic recommendations of 'Who to Follow', likely leading to greater assortative mixing (Chaney, Stewart, \& Engelhardt, 2018).

However, there may be political asymmetry in the extent of homophily in Twitter following networks: of the American users who follow official political accounts, Republicans exhibit higher levels of homophily than Democrats (Boutyline \& Willer, 2017; Colleoni, Rozza, \& Arvidsson, 2014). However, this effect is reversed in larger, non-political following networks, where Democrats display stronger homophily (Colleoni et al., 2014). It remains unknown whether such asymmetry is also present in UK Twitter samples.

Despite these asymmetries, following patterns of American Twitter users have been used in successful binary classification of users as Republican or Democrat (Sylwester \& Purver, 2015), and in creating a continuous unidimensional measure of ideology (Barberá, 
2015), the latter of which strongly correlated with individual voting registration records $(r=.80)$. This study adopted an approach that combined these two.

Firstly, this study created a binary classification of the followers of UK voters as Liberal or Conservative through the ideology of the political elites they follow. To increase the likelihood of political homophily holding true, this study excluded users who followed both Liberal and Conservative elites (as in Barberá, 2015 and Sylwester \& Purver, 2015) - these might be either 'centrist' users or users consciously choosing to expose themselves to alternative opinions. For users who follow both political 'sides', inferring political leaning from following patterns becomes more difficult without the application of unsupervised clustering algorithms, which need their own validation (e.g. Himelboim et al., 2013), and thus go beyond the scope of this study. We note that this exclusion criteria may have led us to exclude accounts that, for example, only followed one elite with opposing views. This all-or-nothing approach may have limited our sample to politically homogeneous users. Nevertheless, the excluded group only constituted a fifth of the initial sample of users, leaving a sufficient number of users in our analysis.

In summary, this study created two directed networks of Twitter following - Liberal (i.e. Left) and Conservative (i.e. Right). Because these networks only involved political connections (i.e. voters following politicians), the presence, amount and quality of network connections were all interpreted as signals of the strength of each voter's political leaning. Specifically, inspired by the USA-based model from Barberá (2015), voters with greater Centrality in their respective network (Newman, 2010) were assumed to have stronger political attitudes than voters less central in the network. This continuous Centrality metric extends the assumption of homophily beyond binary Left-Right labels, imitating the ordinal Likert scale measures used by Cichocka et al. (2016). Not to be confused with political 'centrality', network Centrality measures find the most influential or engaged nodes in a graph by looking at the quantity and quality of their connections. Hubs Centrality (Kleinberg, 1999) was used to create this continuous measure - this assigned higher Centrality values to users who followed a larger number of more popular political elites. It was predicted that users More Central in the Conservative political network would exhibit a stronger preference for Nouns, and More Central Liberal users would use fewer Nouns.

The sampling methodology in this study did not allow for the distinction of Social versus Economic Conservatism, as in Study 1 or in Cichocka et al. (2016). It may have been possible to weigh Twitter's political networks by the strength of Social Conservatism of the 
elites; however, little about this is known at the individual MP level, and we were unable to obtain the data where it is known (Bale, Cheung, Cowley, Wager, \& Menon, 2020).

\section{Remaining concerns about Twitter as a research instrument}

One strong concern that remains is potential population bias on Twitter, as Twitter users tend to be younger, more educated and wealthier than average (Mellon \& Prosser, 2017; Pew Research Center, 2019b). This relates to the largely self-selecting nature of samples from social networking sites, whereby factors such as age, occupation and social ties make certain individuals more likely to use Twitter than others (Hargittai, 2015). These population biases on Twitter are likely to differ from the biases in questionnaire studies or lab-based social experiments, where participants tend to be undergraduate students from Western democracies (Henrich, Heine, \& Norenzayan, 2010). Thus, a combined exploration of language and politics through both Twitter and questionnaires may lead to greater representativity than the use of either method alone.

A further population bias may arise on Twitter in the political leanings of users, with evidence of a Left skew in both the US (Pew Research Center, 2019b) and the UK (Mellon \& Prosser, 2017). This is somewhat evident in our final sample on the Right $(N=16,170)$ being smaller than that on the Left $(N=17,788)$. However, a UK-based sample validation has shown that this political bias may be mediated by Twitter users' demographic characteristics such as age, gender and education (Mellon \& Prosser, 2017). These are likely to act as confounding variables in questionnaire studies too, including the study that this paper set out to replicate (Cichocka et al., 2016), where a Left skew was present in most measures of Conservatism. Nevertheless, another political bias on Twitter is possible - a comparative study in Spain found that users who tweet about politics tend to have more extreme ideological preferences (Barberá \& Rivero, 2014). This bias should have been avoided in this study through the use of 'following' networks in sampling, rather than by finding political tweets. Nevertheless, to eliminate potential Twitter-specific population effects, a validation was performed in Study 3 using a questionnaire and recruiting a politically balanced sample from Prolific.

Another concern regarding Twitter data is the prevalence of fake 'bot' accounts, or machine algorithms that imitate human users. To prevent such accounts from confounding our results, this study applied activity filters from past literature (Barberá, Jost, Nagler, Tucker, \& Bonneau, 2015; Sylwester \& Purver, 2015), excluding suspicious accounts that produced little original content and/or demonstrated a tendency for repeated re-tweeting. 
Finally, major ethical concerns arise with the use of Twitter data, specifically around informed consent and user privacy. Informed consent cannot be obtained by traditional means (i.e. using a signed consent form) from millions of users. However, all users included in this study had non-private Twitter accounts, and have signed a user agreement stating that they consent to make their account details (including their profile descriptions and who they follow) and their tweets publicly available through the API (Twitter, 2020). Nevertheless, unresolved ethical questions remain regarding whether users actually read these agreements before accepting them or when these get updated.

The second key ethical concern in Twitter research is user privacy. Because all tweets used in this study are public, they can be searched on the platform and thus the author of the tweet can be identified. This makes it difficult to ethically publish the collection of tweets in line with Open Science practices. Furthermore, the research reported here infers traits such as political leaning and strength of political engagement about individual users. A recent scandal involving Cambridge Analytica has shown the ease with which others can misuse such personal data for political targeting. To prevent this and comply with the Twitter Developer Agreement and the General Data Protection Regulation (GDPR), individual tweets were not made publicly available. The network dataset and the collection of tags are available upon request.

\section{Summary of this study}

In summary, this study aimed to re-test the association between Conservatism and Noun use in real-world Twitter communication. First, all Twitter followers of British MPs were collected and categorised as Left vs. Right, based on the political affiliation of the MP they follow, creating a grouping variable Side. Then, attempting to create a continuous measure of strength of ideology, two networks of Twitter users were built (Left and Right, with the exclusion of 'centrist' users), and Hubs Centrality scores (Kleinberg, 1999) were calculated for every user within their respective network. Finally, a part-of-speech tagger was applied to the tweets of these users to create the dependent variable Noun use. Content analysis was used to validate all measures.

\section{Method}

\section{Participants and Procedure}

First, a list of political elites was collected, including party leaders, MPs and MEPs who held office in February 2020. Differing from past USA-based explorations (e.g. Barberá, 2015), 
this study did not include media accounts as elites, as these would be more difficult to categorise due to the stricter rules on British media impartiality. To construct this list, the official Parliament website was first (https://members.parliament.uk/members/Commons) to collect Twitter handles for the MPs that had them $(N=382)$. Then, we manually added the '@brexitparty' account, party leaders who were not MPs (e.g. ‘NicolaSturgeon'), and available MEP accounts (as listed on the European Parliament website in 2020, http://www.europarl.europa.eu/meps/en/search). This resulted in a list of 467 elites. Then, in line with existing research (Barberá, 2015), accounts without 'verified' Twitter status and accounts with fewer than 2000 followers were excluded as potential impersonators. Finally, three accounts were excluded for failing to represent any political 'side' - the Speaker for the House of Commons and two 'Independent' MPs.

This left 420 elites, which were labelled as Left or Right based on party affiliation. To avoid confusion with the UK 'Conservative' party, Liberalism and Conservatism, and the corresponding networks, are referred to as 'Left' and 'Right' in this study. There were 7 Left parties (Labour, SNP, Liberal Democrats, Plaid Cymru, Green, Alliance Party of Northern Ireland, Sinn Féin) and 3 Right parties (Conservatives, Brexit Party, Democratic Unionist Party). Next, user IDs from all Twitter followers of the 420 elites $(N=8,036,809$ unique users) were collected using the Twitter REST API - during this, followers of only 418 elites were returned by the API, likely due to the deletion of two elites' accounts during data collection. The final sample of elites contained 227 Left and 191 Right accounts (full list available at (https://osf.io/dr7bk/?view only=27f913c49c7b48019484f784b5db4135).

Subsequently, this sample is considered through a network approach, where each node is stored as the user ID of either a voter or a political elite, and each directed edge between two nodes represents who follows who on Twitter. Because the sample was collected based on the following of elites, the only possible directions of each edge are: from a voter to an elite, as in the majority of cases, or from one elite to another, if elite A was listed in the followers of elite B. To re-iterate, information about elites following voters was not recorded, as was the data about voters following each other. Hence, this study did not look at entire naturally emerging Twitter communities, but only at political interactions of voters with elites.

After collecting the $8 \mathrm{mln}$ followers of all elites, each voter in this directed network was labelled as Left and/or Right, based on the party affiliation of the elites they followed. Throughout the remainder of this study, capitalised terms 'LEFT' and 'RIGHT' are used to describe the two political networks. Most users followed more than one elite, and naturally, 
some users also followed both LEFT and RIGHT elites $(N=1,517,567)$ - as discussed, these users were considered politically 'centrist' or otherwise complex and were excluded to allow us to rely on the homophily hypothesis (as in Barberá, 2015, and Sylwester \& Purver, 2015). After excluding the centre, two groups of users remained - LEFT $(N=3,853,285)$ and RIGHT $(N=2,676,014)$. The exclusion of 'centrist' voters and elites created some disconnected components on the graph. Using common procedures from Network Science, the largest weakly connected component was extracted from each side. This resulted in a LEFT graph with $N=822,781$ nodes, of which 29 were elites, and 1,019,598 edges between these nodes; and a RIGHT graph with $N=1,542,256$ nodes, of which 35 were elites, and 1,840,621 edges between these nodes. The creation of the LEFT and RIGHT graphs is illustrated in Figure 3 below.

Step 1. Collect all Twitter followers of $418 \mathrm{UK}$ political elites (MPs, MEPs and Party accounts). The elites were labelled as LEFT or RIGHT based on their party identity.
Step 2. Delete voters who followed both LEFT and RIGHT elites.

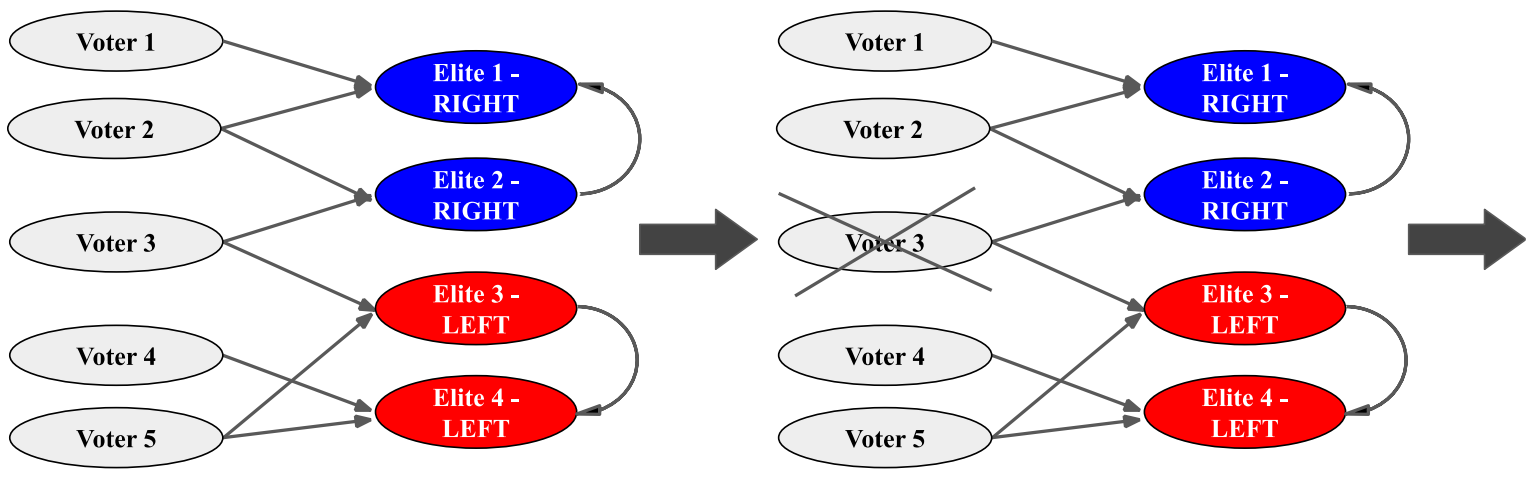

Step 3. Label the remaining voters as LEFT or The Result: Two graphs, LEFT and RIGHT RIGHT, based on the label of the elite(s) they follow.

Our Sample Our Elites

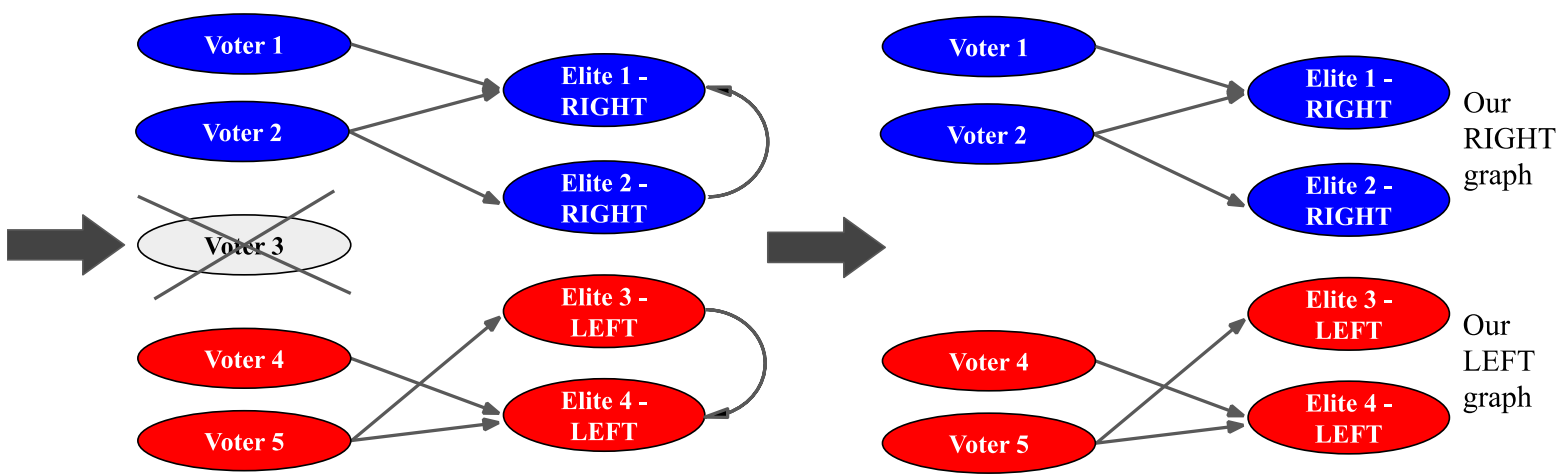

Figure 3. Schematic representation of how the LEFT and RIGHT Twitter Networks were built. Colour represents political side $($ Red $=L E F T$, Blue $=$ RIGHT $)$. 
Then, due to time limits and computational limits, two sets of 100,000 non-elite users were randomly sampled from the LEFT and the RIGHT graphs, and tweets from these users were collected using the Twitter API, between $23^{\text {rd }}$ and $27^{\text {th }}$ March 2020, using the tweepy package in Python. Due to API limits, time limits and user privacy settings, it was only possible to obtain up to roughly 200 most recent tweets from each user, and only from public accounts. This resulted in 19,739,100 tweets overall, 10,603,262 from 79,261 LEFT users, and 9,135,838 from 76,931 RIGHT users. These tweets were stored in a MongoDB database.

Subsequently, activity filters were applied for each non-elite user, informed by previous work in the field. As in Barberá (2015), this study discarded users who 1) had sent fewer than 100 tweets overall, 2) had under 25 followers, 3) were not tweeting in English (based on Twitter's automatic language detection), and 4) had not sent a tweet in the past six months. It was impossible to filter by location (as in Barberá, 2015) because Twitter no longer makes tweet geolocation data available through the API, and only a few users list their location in their profile descriptions. Then, elites' tweets were also excluded. Finally, during the creation of the network centrality metric (described subsequently), the algorithm failed to return values for 326 RIGHT users - these therefore also had to be excluded. Thus, the main analysis was carried out with 17,788 LEFT and 16,170 RIGHT users ( $N=33,958$ overall), and 3,090,937 LEFT and 2,280,722 RIGHT tweets. For the network centrality analysis (described below), the elites originally associated with these users were also considered - there were 29 LEFT elites and 35 RIGHT elites. The two groups were not reduced to equal sizes in the main analysis to avoid invalidating the network centrality metrics, as network centrality is based on all actors in a network.

Due to the nature of this study, specific demographic characteristics of the sample remain unknown. However, estimates suggest that an age range between 18 and 49 makes up between 70\% (in the US, Pew Research Center, 2019a) and 79\% (globally, Statista, 2020) of the overall Twitter population. The estimated gender split on Twitter in the US is $50 \%$ female and 50\% male (Pew Research Center, 2019b), although users that tweet actively about politics are more likely to be majority women (65\% of the top $10 \%$ most active political tweeters are women; Pew Research Center, 2019b). As we were unable to filter by geographical location, nationality and country of residence may also be potential confounds - it is likely that some users in our networks, despite tweeting in English, were not UK nationals or residents. 


\section{Materials}

\section{Noun score:}

To create the dependent variable Noun use, a pre-trained part-of-speech (POS) tagger titled TweetNLP (Owoputi et al., 2013) was applied to the tweets of LEFT and RIGHT users. This tool assigned a POS tag (e.g. Noun, Verb, etc.) to every word in a tweet, based on both the word itself and its grammatical context. Tags in this tool are classified into five categories (see Appendix $D$ for the full classification and examples):

1. Nominal (Common Noun, Pronoun, Nominal + possessive, Proper Noun, Proper Noun + possessive, Nominal + verbal, and Proper Noun + verbal)

2. Other open-class words (Verb, Adjective, Adverb, Interjection)

3. Other closed-class words (Determiner, Pre/Post-position or subordinating conjunction, Coordinating conjunction, Verb particle and others)

4. Twitter/online-specific (hashtag “\#”, “@”-mention, discourse markers “RT” and “:” in retweets, URL or email address, and Emoticon)

5. Miscellaneous (numeral, punctuation and other)

For each user in the sample, we set out to calculate average proportions of Noun tags per tweet, so these could be compared between the two sides. Nouns in every tweet were counted as the sum of tags 'Noun' (e.g. "book”), 'Nominal + possessive' (e.g. "books'”) and 'Nominal + verbal' (e.g. "book'll”). This is slightly problematic, however, as the second and third of these groups also include Pronouns (e.g. "he'll”). The influence of this was examined in more detail using content analysis.

The general formula for calculating the Noun proportion in a single tweet was as follows:

$$
\text { Noun proportion }=\frac{\# \text { Noun tags }}{\text { total } \# \text { tags }},
$$

where '\#' indicates 'number of'. This formula calculates the Noun proportion in each tweet. We calculated this for every tweet from every user, and then computed a mean_proportion score as the average of all Noun proportion scores for that user.

Since the finding that inspired this study originated from a questionnaire (see Cichocka et al., 2016), rather than from online speech, a second, more controlled proportion score was created. Here, Twitter-specific or otherwise special characters which are not a part of the meaningful sentence were excluded from the denominator. Special characters were defined as hashtags (“\#”), “@”-mentions, discourse markers ( “RT” and “:” in retweets), URLs or email addresses, Emoticons and punctuation. Had these special characters not been excluded from the calculation, the discovered effects might have been confounded with other differences, such as the LEFT using more Emoticons in tweets in general (Wojcik et al., 2015). Notably, 
numerals (e.g. "2010”) and other abbreviations (e.g. "wby” for "what about you”) were kept in the denominator in this score, as these could still constitute a meaningful part of the sentence. Thus, the second score, proportion_filtered, was created as follows:

$$
\text { Noun proportion filtered }=\frac{\# \text { Noun tags }}{\text { total } \# \text { tags }-\# \text { special charactres }} \text {. }
$$

Finally, some uncertainty remained as to whether users on the LEFT and RIGHT might use closed-class words (such as Determiners, e.g. "the”) differently. To eliminate potential influences of such differences, a third, even more strict measure of Noun proportion was created: open_proportion. This was the number of Nouns divided by the number of only openclass words (i.e. only Nouns, Verbs, Adjectives, Adverbs, Interjections), as follows:

$$
\text { Noun open proportion }=\frac{\# \text { Noun tags }}{\# \text { open class words }} \text {. }
$$

For purposes of statistical multi-versing (Steegen, Tuerlinckx, Gelman, \& Vanpaemel, 2016), all three proportion scores were considered throughout the analysis.

As noted, the POS tagger distinguished between Common Nouns and Proper Nouns. Since Proper Nouns were not investigated in the original study by Cichocka et al. (2016), these were considered in a separate score, distinct from Common Nouns. Proper Nouns in a tweet were counted as the sum of tags 'Proper Noun' (e.g. "iPad”), 'Proper Noun + possessive' (e.g. "America's") and 'Proper Noun + verbal' (e.g. "Mark'll”). Proper Noun counts were not confounded with any other part-of-speech in the TweetNLP classification.

The three scores were calculated for Proper Nouns the same way as for Common Nouns. For clarity, throughout the rest of this paper, 'Common Nouns' refers to nouns as in Cichocka et al.'s (2016) study, and proper nouns are referred to as 'Proper Nouns'. When talking about both groups in general, we use the capitalised term 'Nouns'.

In summary, two separate sets of three Noun scores were created for each tweet, listed below from the most constrained (open_proportion) to the least (proportion):

$$
\begin{aligned}
& \text { Common Noun open proportion }=\frac{\# \text { Common Noun tags }}{\# \text { open class words }} \\
& \text { Common Noun proportion filtered }=\frac{\# \text { Common Noun tags }}{\text { total \# tags }-\# \text { special charactres }} \\
& \text { Proper Noun open proportion }=\frac{\# \text { Common Noun tags }}{\text { total } \# \text { tags }} \\
& \text { open } \text { class } \text { words } \text { tags }
\end{aligned}
$$




$$
\begin{aligned}
& \text { Proper Noun proportion filtered }=\frac{\# \text { Proper Noun tags }}{\text { total \# tags }-\# \text { special charactres }} \\
& \text { Proper Noun proportion }=\frac{\# \text { Proper Noun tags }}{\text { total } \# \text { tags }}
\end{aligned}
$$

These were then averaged across all of a user's tweets to create mean_proportion, mean_proportion_filtered and mean_open_proportion scores for Common and Proper Nouns respectively.

To validate the POS tagger, 30 of the most frequently used Common and Proper Nouns on the LEFT and RIGHT were analysed. As is common in Natural Language Processing (e.g. Sylwester \& Purver, 2015), during this word frequency analysis all the words were first put to lowercase, and filtering was applied to get rid of various endings on words (so that, for example, "Sunday" and "sunday's" would be grouped as the same word; see Appendix E for detail). The filtering was particularly necessary to extract the plain Noun from the '...+ possessive' and '... + verbal' categories.

The results of this word frequency analysis (presented in Figures 4 and 5 below, and further in Appendix E) illustrated the problem highlighted earlier: some Pronouns (e.g. "I", "it”) were included in the Common Nouns tags, because the TweetNLP classification grouped them together in the 'Nominal + possessive' and 'Nominal + verbal' categories. The results of the content analysis also highlighted an unexpected problem - some Emoticons (or 'Emoji') were incorrectly classified as Proper Nouns (Figure 5). 

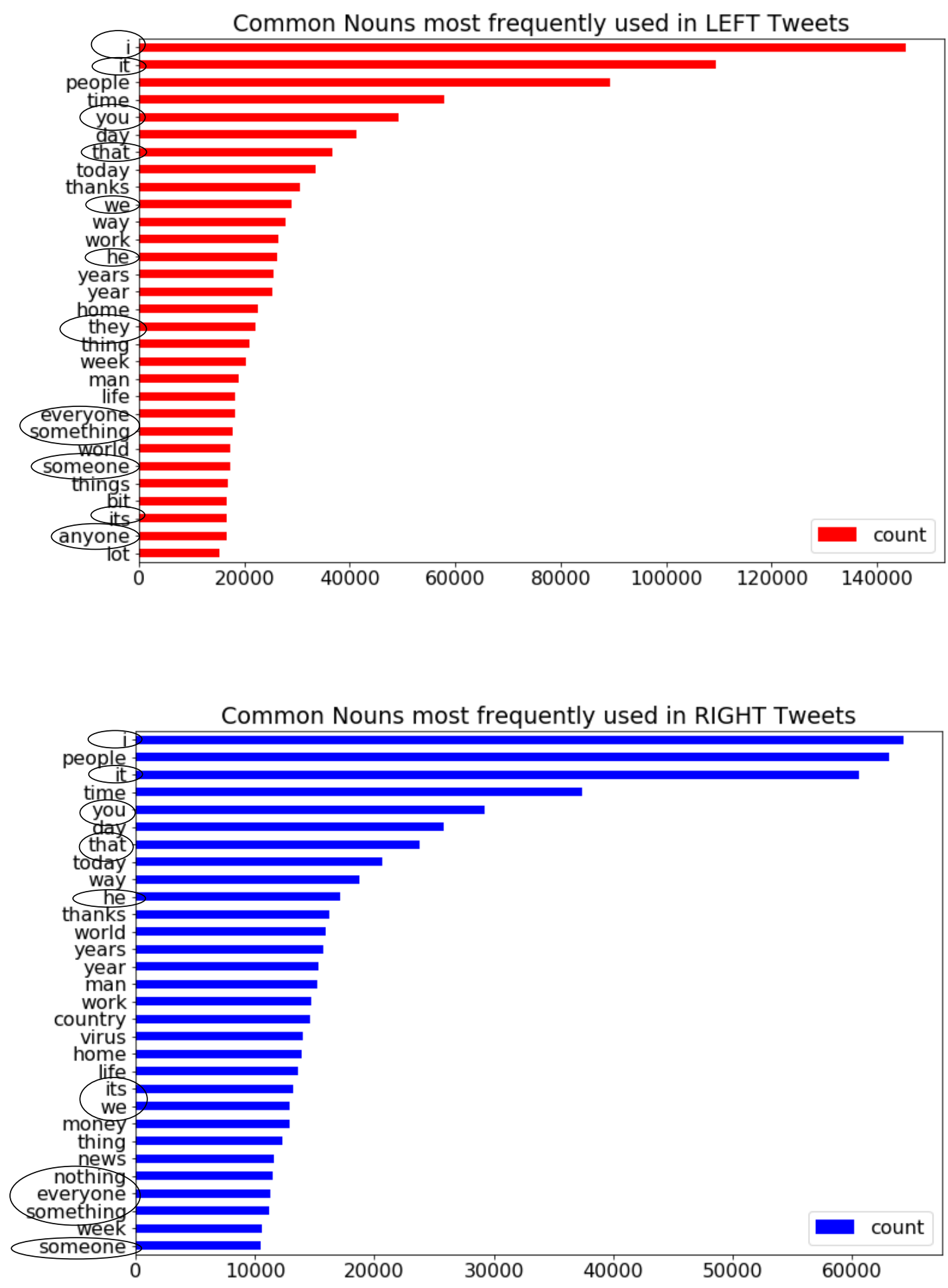

Figure 4. 30 most frequently used tags labelled as Common Nouns in tweets, on the LEFT (red) vs. RIGHT (blue). The circled words represent mis-classified Pronouns. A cleaned version of this diagram is available in Appendix E. 

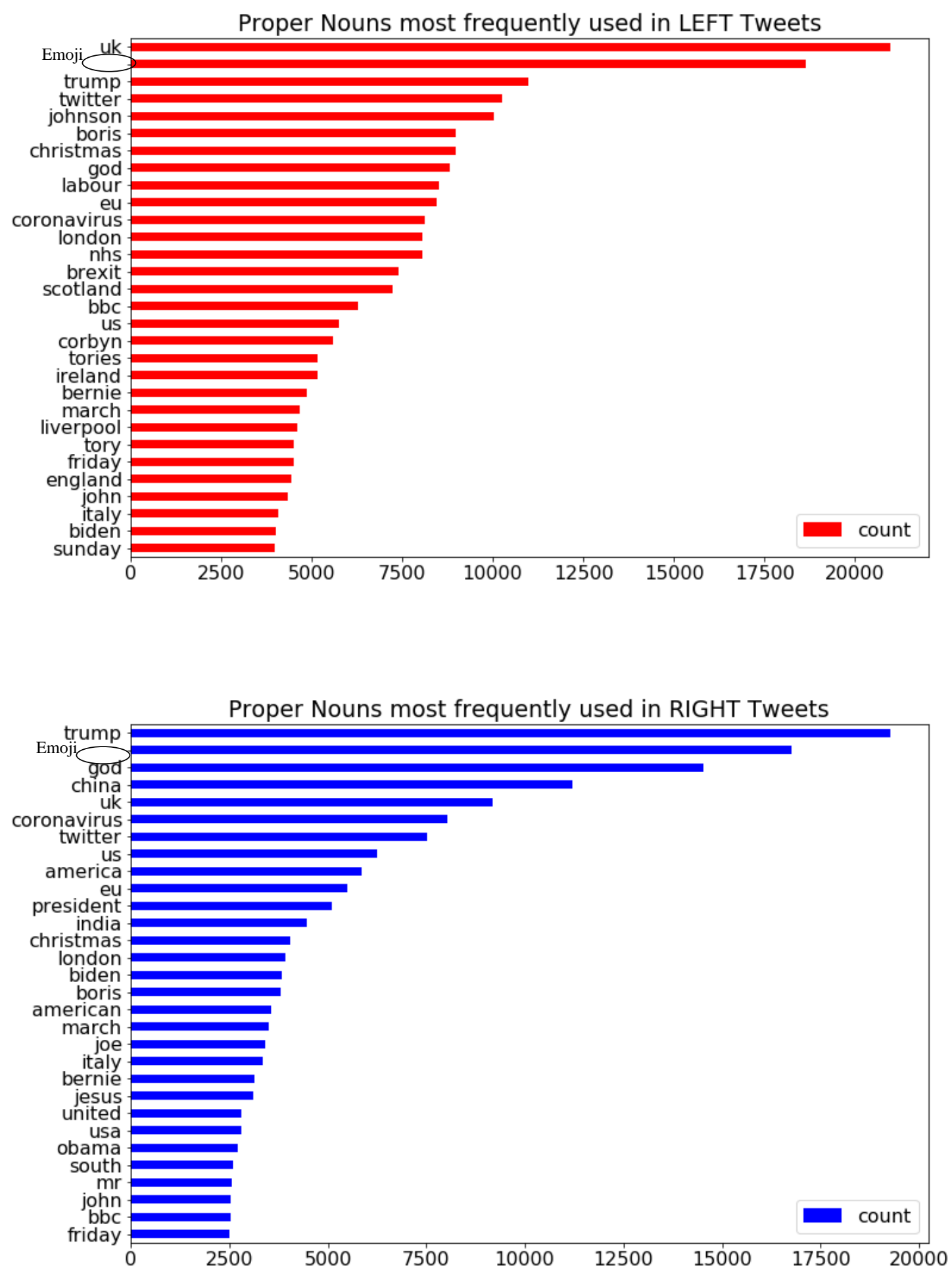

Figure 5. 30 most frequently used tags labelled as Proper Nouns in tweets, on the LEFT (red) vs. RIGHT (blue). The circled blank represents a group of mis-classified Emoticons/Emoji. A cleaned version of this diagram is available in Appendix E.

To investigate Pronouns mis-classified as Common or Proper Nouns, a list of 94 Pronouns was created manually (see Appendix E), and string matching was used to find these 
in the two lists of Nouns. This study set out to exclude mis-classified tags if they made up more than $10 \%$ of the category of interest ${ }^{1}$.

The results of string matching showed that Pronouns constituted $9.8 \%$ of our Common Nouns and $0.6 \%$ of our Proper Nouns. Thus, all Pronoun tags were excluded from the Common Nouns list before proceeding. The reported results for Proper Nouns include the $0.6 \%$ Pronoun tags, but results remained robust to removing Pronouns from the Proper Nouns list too (as reported in Appendix K).

Repeating the procedure for Emoticons, these were flagged in our collections of Nouns using Unicode values. Emoticons constituted 1\% of our Common Nouns and $1.2 \%$ of our Proper Nouns. As this did not surpass the 10\% threshold, Emoticons were kept in the data; however, the results were also robust to removing these (see Appendix $K$ ). Word frequency graphs for cleaned sets of Common and Proper Nouns are available in Appendix E.

Finally, as the Tweets were collected in March 2020, variations of the word 'coronavirus' may have constituted a large proportion of our Common or Proper Nouns. This would have been problematic for two reasons. First, the POS tagger, developed in 2013, did not correctly and consistently label variations of the relatively novel word 'Coronavirus' sometimes these were labelled as Common and sometimes as Proper Nouns. This may be caused by inconsistencies in the use of capital letters (Owoputi et al., 2013), and ambiguities in definition - dictionary entries suggest that 'coronavirus' can be used as either a Common Noun (describing a group of coronaviruses, of which there are more than one), or as a Proper Noun describing Covid-19 specifically (Merriam-Webster., n.d.). Second, it was unclear whether, in the context of a global emergency such as Covid-19, people would change their linguistic habits, and how this interacts with political preferences - for example, perhaps the tendency of the RIGHT to react more strongly to negative events (Hibbing, Smith, \& Alford, 2014) or to perceive the world as a dangerous place (Duckitt, Wagner, Du Plessis, \& Birum, 2002; van Leeuwen \& Park, 2009) might cause them talk more about Covid-19 online than the LEFT, artificially increasing the difference in Noun scores. To check whether this would be a significant problem, a list of 23 'Coronavirus' words was compiled manually (see Appendix $E$ ), and proportions were calculated of these words in our collections of Common and Proper Nouns. This showed that 'Coronavirus' words only constituted $0.1 \%$ of all Common Nouns,

\footnotetext{
${ }^{1}$ This $10 \%$ threshold criterion was defined a-priori in conversation with Professor Mark Haggard at the University of Cambridge.
} 
and $0.9 \%$ of all Proper Nouns. Thus, the reported results include these words; yet, the results also remained robust to their exclusion (see Appendix K).

To summarise, after the filtering of users and Noun tags, the final sample consisted of a total of 5,371,659 tweets from 33,958 users. Only original tweets were considered - all retweets were excluded from analysis. On average, participants' tweets were made up of $15.4 \%$ Common Nouns $(S D=4.5 \%)$ and $6.2 \%$ Proper Nouns $(S D=5.6 \%)$ per tweet (mean_proportion); $21.0 \%$ Common Nouns $(S D=7.3 \%)$ and $9.0 \%$ Proper Nouns $(S D=9.9 \%)$ per tweet without special characters (mean_proportion_filtered); and 28.9\% Common Nouns per tweet $(S D=9.1 \%)$ and $11.5 \%$ Proper Nouns $(S D=11.2 \%)$ per meaningful POS tags in the tweet (mean_open_proportion).

Descriptive statistics for the three types of Common and Proper Noun scores separated by political Side are presented in Table 2 below. Again, these are reported from the most controlled score (mean_open_proportion) to the least (mean_proportion). It is evident that both scores are strongly leptokurtic (Kurtosis $>9.0$ for all), and a strong positive skew is also present in all of these scores, more so for Proper Nouns than Common Nouns.

\begin{tabular}{|c|c|c|c|c|c|c|c|c|}
\hline DV & Score & Side & $M$ & $S D$ & Median & Range & Skew & Kurtosis \\
\hline \multirow{6}{*}{$\begin{array}{l}\text { Common } \\
\text { Noun use }\end{array}$} & \multirow{2}{*}{$\begin{array}{l}\text { mean_open } \\
\text { - proportion }\end{array}$} & LEFT & $29 \%$ & $7 \%$ & $29 \%$ & $0-100 \%$ & 1.15 & 15.14 \\
\hline & & RIGHT & $28 \%$ & $11 \%$ & $28 \%$ & $0-100 \%$ & 1.58 & 11.46 \\
\hline & \multirow{2}{*}{$\begin{array}{l}\text { mean }_{\text {proportion }} \\
\text { filtered }\end{array}$} & LEFT & $21 \%$ & $5 \%$ & $21 \%$ & $0-100 \%$ & 2.37 & 34.31 \\
\hline & & RIGHT & $21 \%$ & $9 \%$ & $21 \%$ & $0-100 \%$ & 3.00 & 23.84 \\
\hline & \multirow{2}{*}{$\begin{array}{c}\text { mean }_{-} \\
\text {proportion }\end{array}$} & LEFT & $16 \%$ & $3 \%$ & $16 \%$ & $0-67 \%$ & 0.41 & 11.49 \\
\hline & & RIGHT & $15 \%$ & $5 \%$ & $15 \%$ & $0-75 \%$ & 0.65 & 8.48 \\
\hline \multirow{6}{*}{$\begin{array}{c}\text { Proper } \\
\text { Noun use }\end{array}$} & \multirow{2}{*}{$\begin{array}{l}\text { mean_open } \\
\text { - proportion }\end{array}$} & LEFT & $10 \%$ & $8 \%$ & $8 \%$ & $0-100 \%$ & 4.29 & $32.30-$ \\
\hline & & RIGHT & $13 \%$ & $14 \%$ & $9 \%$ & $0-100 \%$ & 3.55 & 16.43 \\
\hline & \multirow{2}{*}{$\begin{array}{l}\text { mean- } \\
\text { proportion } \\
\quad \text { filtered }\end{array}$} & LEFT & $8 \%$ & $6 \%$ & $6 \%$ & $0-100 \%$ & 5.56 & 54.55 \\
\hline & & RIGHT & $10 \%$ & $12 \%$ & $7 \%$ & $0-100 \%$ & 4.21 & 23.03 \\
\hline & \multirow{2}{*}{$\begin{array}{c}\text { mean }_{-} \\
\text {proportion }\end{array}$} & LEFT & $5 \%$ & $4 \%$ & $5 \%$ & $0-71 \%$ & 4.02 & 31.09 \\
\hline & & RIGHT & $7 \%$ & $7 \%$ & $5 \%$ & $0-83 \%$ & 3.54 & 20.02 \\
\hline
\end{tabular}

Table 2. Descriptive Statistics for the two groups and three versions of the dependent variable (mean Noun-per-tweet proportions), separated by Side. 
Side:

A categorical between-subjects variable Side with two levels (LEFT vs. RIGHT) was created from Twitter following patterns, as described in the Participants and Procedure section. The final sample consisted of 17,788 LEFT and 16,170 RIGHT users.

\section{Network Centrality:}

Beyond political Side, this study also aimed to use network Centrality measures to infer the strength of each user's political affiliation from the Twitter networks. In a directed network, there are three main ways of measuring Centrality: Out-Degree Centrality, PageRank and Hubs/Authorities (Newman, 2010). Hubs/Authorities (Kleinberg, 1999) was chosen because, unlike PageRank (Brin \& Page, 1998), it considered outgoing as well as incoming links, and was less likely than Out-Degree Centrality to be extremely positively skewed (Barabási \& Albert, 1999). As illustrated in Figure 6 below, the Hubs/Authorities algorithm divides the network into two types of nodes - Hubs, who point towards many nodes that are also popular with other Hubs, and Authorities, who are pointed at by many nodes who also point at other Authorities. The Hubs-Authorities dichotomy maps nicely onto our network of voters and elites - the algorithm should flag active voters, who follow many popular elites (i.e. Authorities), as nodes with greater Hubs scores. The scores were calculated using the HITS algorithm in the Python NetworkX package (Hagberg, Schult, \& Swart, 2008). Descriptive statistics are presented in Appendix $H$.
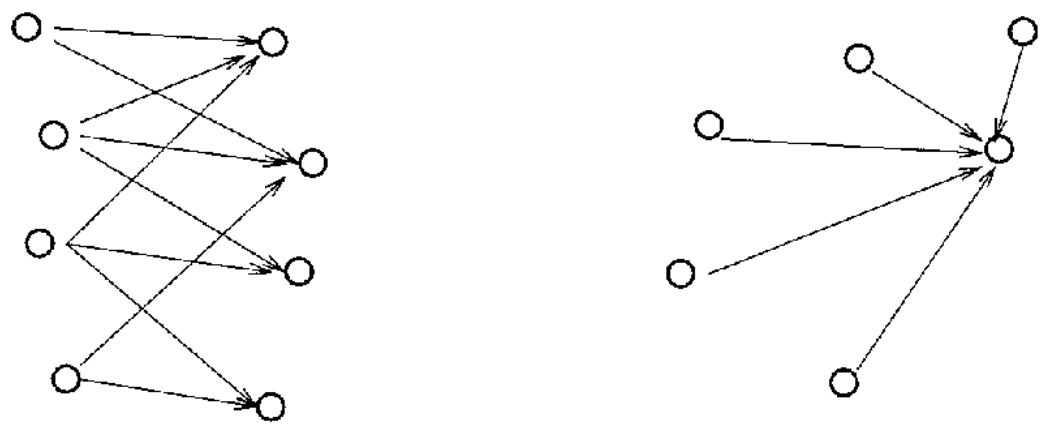

unrelated page

of large in-degree

hubs

authorities

Figure 6. An illustration of a densely linked set of Hubs and Authorities versus an unrelated page of large in-degree. Borrowed from Kleinberg (1999).

To validate Hubs Centrality specifically as a measure of strength of political leaning, this study used content analysis of extreme cases. This involved an automated comparison of the profile descriptions of 100 most and 100 least Hubs-central users on the LEFT versus the 
RIGHT. In line with this study's Ethics principles and the Twitter Developer Agreement, this analysis was performed in aggregate, for each set of 100 users together, making it impossible to distinguish entries from individual users.

The results of this analysis, presented in full in Appendix $F$, suggested that Hubs Centrality was a valid proxy for strength of political engagement - the profiles of 100 most central users on both sides did involve a lot of political content such as party membership (e.g. "labour", "conservative”) and political topics (e.g. "brexit”). However, this analysis did not clearly demonstrate that users with higher Centrality would indeed have stronger ideology these profiles did not contain any far-Right or far-Left terms. Thus, our Centrality measure likely demonstrates strength of involvement in politics for that specific side, but not necessarily the strength or ideology. This distinction between engagement and ideology has been suggested for both self-reported political ideology (Abramowitz, 2010) and for automatic prediction from Twitter posts (Preoţiuc-Pietro et al., 2017). As noted earlier, the centrality metric was also not normalised for the amount of 'friends' of a user, potentially putting the validity of the whole metric to question.

One more robustness check was performed with the Centrality measure. The measures reported above were calculated based on the entire, unfiltered networks (800K LEFT and $1.5 \mathrm{mln}$ RIGHT nodes), yet the Noun proportions were only considered for the filtered sample of $17 \mathrm{~K}$ LEFT and 16K RIGHT users. Thus, the resulting Hubs scores represent Centrality in a group of people larger than the one used for analysis of Noun use. Nevertheless, a re-calculation of the Centrality scores within the smaller graphs only (i.e. 17K LEFT plus the relevant elites, 16K RIGHT plus elites) resulted in similar distributions (see Appendix $G$ ). Thus, the original Hubs scores from the two large graphs were used throughout the rest of the analysis.

The distributions of Hubs scores on the LEFT and the RIGHT appeared to be strongly bimodal (see histograms in Figure 7 below, and skew and kurtosis values in Appendix $H$ ). In practice, bimodality in Hubs scores would suggest that Twitter users may fall into two types those very actively engaged in the political networks and following many popular political elites, and those less engaged. Two methods were used in conjunction to statistically test for non-unimodality (Pfister, Schwarz, Janczyk, Dale \& Freeman, 2013): the Bimodality Coefficient, compared against a benchmark value of 0.55 , with higher numbers pointing toward bimodality (Freeman \& Dale, 2013), and Hartigan's dip statistic (HDS, Hartigan, 1985), which suggests non-unimodality when statistically significant.

The bimodality statistics, descriptive statistics and histograms for the distributions of Hubs scores are presented in Appendix $H$. The two sets of results converged, both pointing 
towards bimodality in the distribution of Hubs scores in the main $35 \mathrm{~K}$ sample. There was also evidence for non-unimodality in the two unfiltered 100K samples, and in the overall original sample of 800K LEFT users and 1.5mln RIGHT users - this suggests that the bimodality in distributions was not an artefact of the random sub-sampling or the activity filtering used. Interestingly, as shown in Appendix $H$, in all of these samples the $B C$ suggested slightly stronger bimodality on the LEFT than the RIGHT.

Due to such strong and consistent evidence for bimodality, a categorical variable for Hubs centrality was created, with two groups: Less vs. More Central. A visual inspection of the histograms (see Figure 7) suggested that a Mean split $\left(M_{\text {Left }}=1.06 \mathrm{e}-06, M_{\text {Right }}=6.94 \mathrm{e}-07\right)$ was better able to separate the two groups than a Median split (Median Left $=4.03 \mathrm{e}-07$, MedianRight=8.34e-07). Thus, users with Hubs scores below the Mean for that side were included in the Less Central group $(N=14,276)$, and users with scores above the Mean for that side were included in the More Central group $(N=19,682)$. The new grouped sample size is presented in Table 3 - these groups ended up being rather imbalanced.
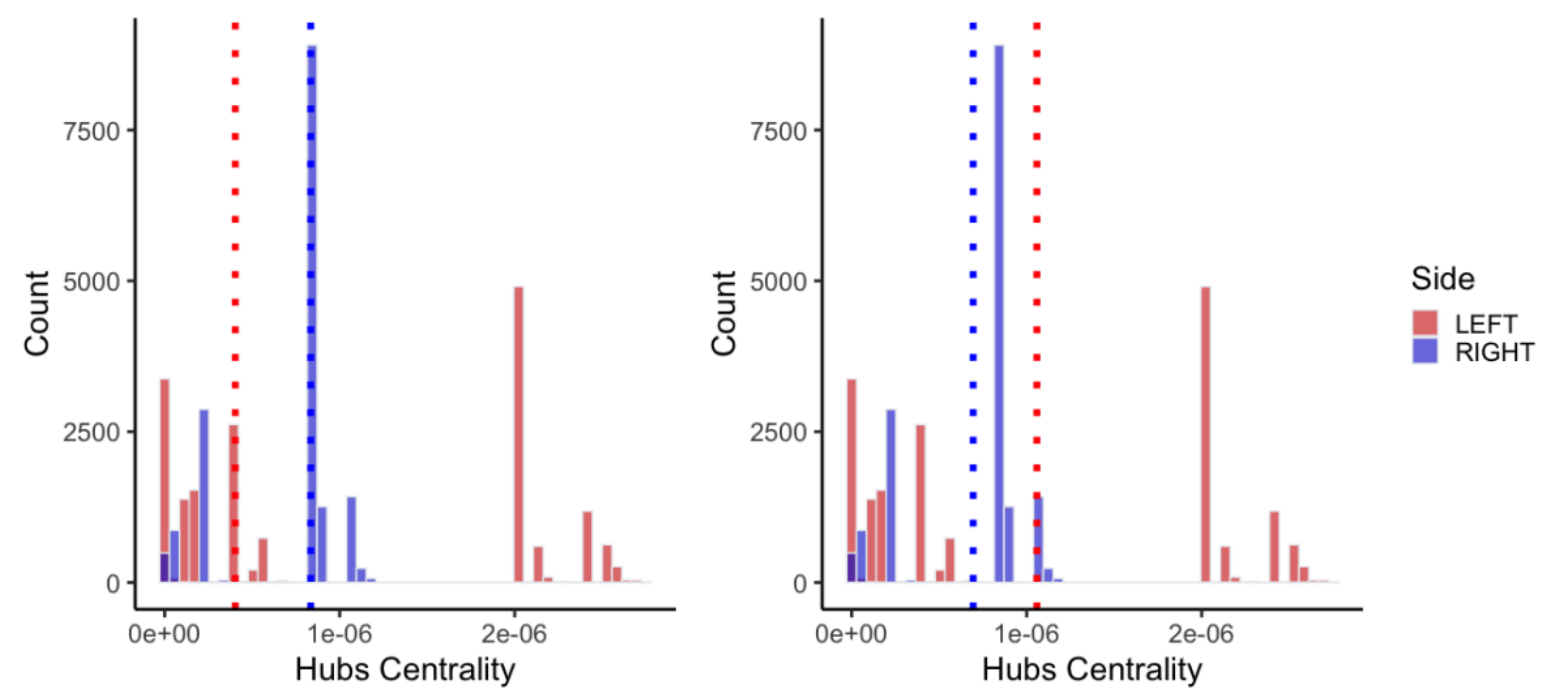

Figure 7. Histogram of Hubs scores plotted by political Side (red=LEFT, blue= RIGHT), illustrating Median (left image) vs. Mean (right image) split using dashed vertical lines.

\begin{tabular}{c|ll} 
& LEFT & RIGHT \\
\hline More central & $N=7783$ & $N=11899$ \\
Less central & $N=10005$ & $N=4271$ \\
Table 3. Grouped sample size by political Side and Centrality group
\end{tabular}




\section{Summary of Design}

To summarise, this study used an independent samples design with one outcome variable Noun use, separated into two types - Common Nouns and Proper Nouns, and three ways of measuring each type - mean_open_proportion, mean_proportion, and mean_proportion_filtered. There were two grouping variables - political Side (with two between-subjects levels - LEFT versus RIGHT) and Hubs Centrality within that side (with two between-subjects levels - Less versus More Central).

\section{Results}

To simultaneously assess the effects of the political Side and network Centrality within that Side on Noun proportions per tweet, two sets of Analysis of Variance (ANOVA) tests were performed - one for Common Nouns (confirmatory) and one for Proper Nouns (exploratory). For each set, three separate models were built - each with a different measure of the dependent variable Noun use (mean_open_proportion, mean_proportion_filtered and mean_proportion). This resulted in two sets of three comparisons, which were corrected using Holm-Bonferroni correction, ultimately dividing by six. Only the adjusted $p$-values are reported.

Due to the imbalance in the sizes of groups, ANOVAs using Type III sums of squares were run (using the car package in $R$ ) instead of the traditional Type I models. Furthermore, to ensure that the direction of the effects in the main ANOVAs was not confounded by the imbalance in group sizes, all ANOVAs were re-run five times on randomly selected samples of $N=4,271$ in each group, using Type I sums-of-squares. The results of these are reported in Appendix I. Finally, the assumption of homogeneity of variance was not met in any of the six tests according to Levene's test; however, this may have been an artefact of the large sample, and there was no strong correlation between the residuals and the fitted values (see plots in Appendix J), so no transformations or heteroskedasticity adjustments were applied. The results are first presented individually for each model, and then summarised in Table 4. The corresponding group means are reported in Table 2.

\section{Common Nouns, as predicted by Side and Centrality}

\subsection{Mean_open_proportion}

The results of a two-way (2x2) ANOVA, illustrated in Figure 8, indicated a statistically significant main effect of Side $\left(F(1,33954)=15.38, p<1.20 \mathrm{e}-15, \eta_{p}{ }^{2}=0.0005\right)$ : on average, Common Nouns represented a higher mean_open_proportion of tweets by LEFT users than 
RIGHT users. There was a significant main effect of Centrality $(F(1,33954)=9.90, p=.012$, $\left.\eta_{p}^{2}=0.0003\right)$, and no interaction effects $\left(F(1,33954)=0.11, p>.999, \eta^{2}=0.000003\right)$.

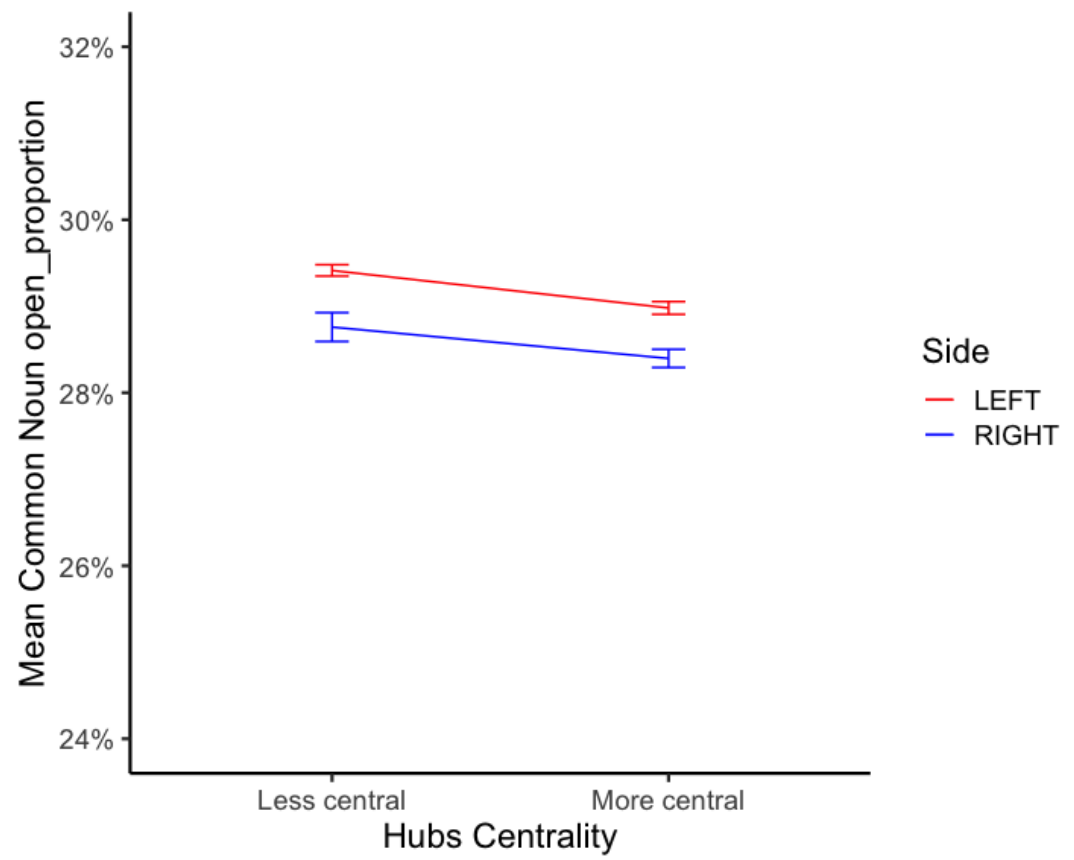

Figure 8. Results of the ANOVA predicting Mean_open_proportion of Common Nouns per tweet.

\subsection{Mean_proportion_filtered}

The results of a two-way ANOVA, illustrated in Figure 9, indicated no main effect of Side $\left(F(1,33954)=0.07, p=.792, \eta_{p}^{2}=0.000002\right)$ or Centrality $(F(1,33954)=4.84, p=.112$, $\left.\eta_{p}{ }^{2}=0.0001\right)$ on mean_proportion_filtered of Common Nouns per tweet per user. There was also no significant interaction effect $\left(F(1,33954)=0.08, p>.999, \eta_{p}^{2}=0.000002\right)$.

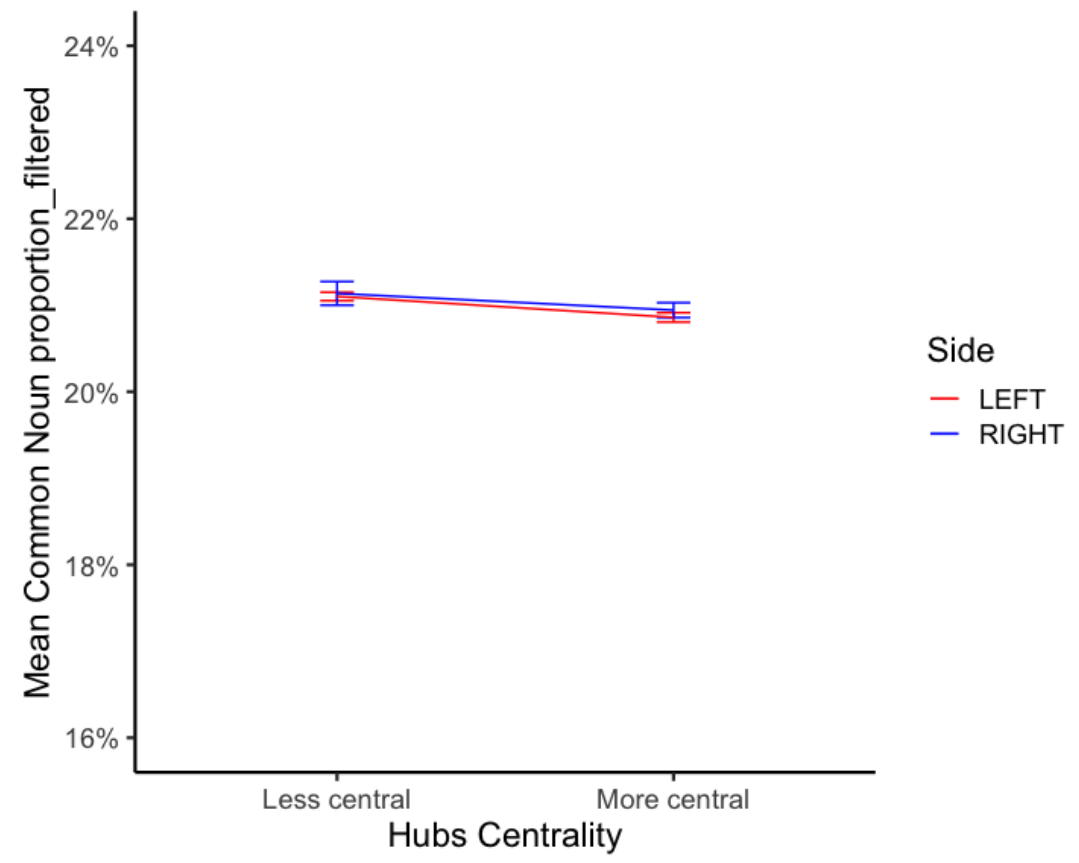

Figure 9. Results of the ANOVA predicting Mean_proportion_filtered of Common Nouns per tweet. 


\subsection{Mean_proportion}

The results of a two-way ANOVA, illustrated in Figure 10, indicated a significant main effect of Side $\left(F(1,33954)=65.39, p<1.20 \mathrm{e}-15, \eta_{p}{ }^{2}=0.002\right)$ : on average, Common Nouns made up a greater mean_proportion of tweets by LEFT users than RIGHT users. There was no significant main effect of Centrality $\left(F(1,33954)=0.76, p=.385, \eta_{p}{ }^{2}=0.00002\right)$, and a marginally significant interaction $\left(F(1,33954)=6.45, p=.066, \eta_{p}{ }^{2}=0.0002\right)$.

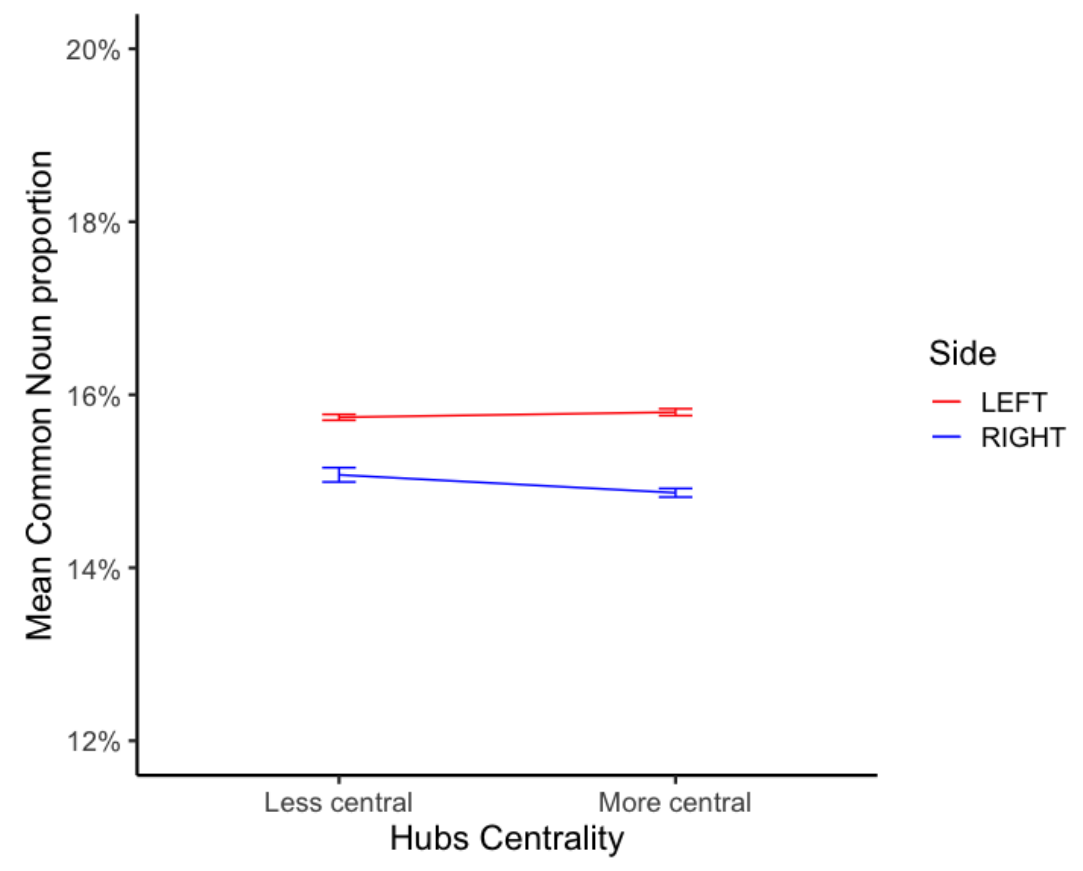

Figure 10. Results of the ANOVA predicting Mean_proportion of Common Nouns per tweet.

\section{Proper Nouns, as predicted by Side and Centrality}

\subsection{Mean_open_proportion}

The results of a two-way $(2 \times 2)$ ANOVA, are illustrated in Figure 11, indicated a statistically significant main effect of Side $\left(F(1,33954)=241.08, p<1.20 \mathrm{e}-15, \eta^{2}=0.007\right)$ : on average, Proper Nouns made up a higher mean_open_proportion of tweets by RIGHT users than for LEFT users. There was no significant main effect of Centrality $(F(1,33954)=5.82$, $\left.p=.080, \eta_{p}{ }^{2}=0.0002\right)$, and no interaction $\left(F(1,33954)=0.02, p>.999, \eta_{p}{ }^{2}=0.0000005\right)$. 


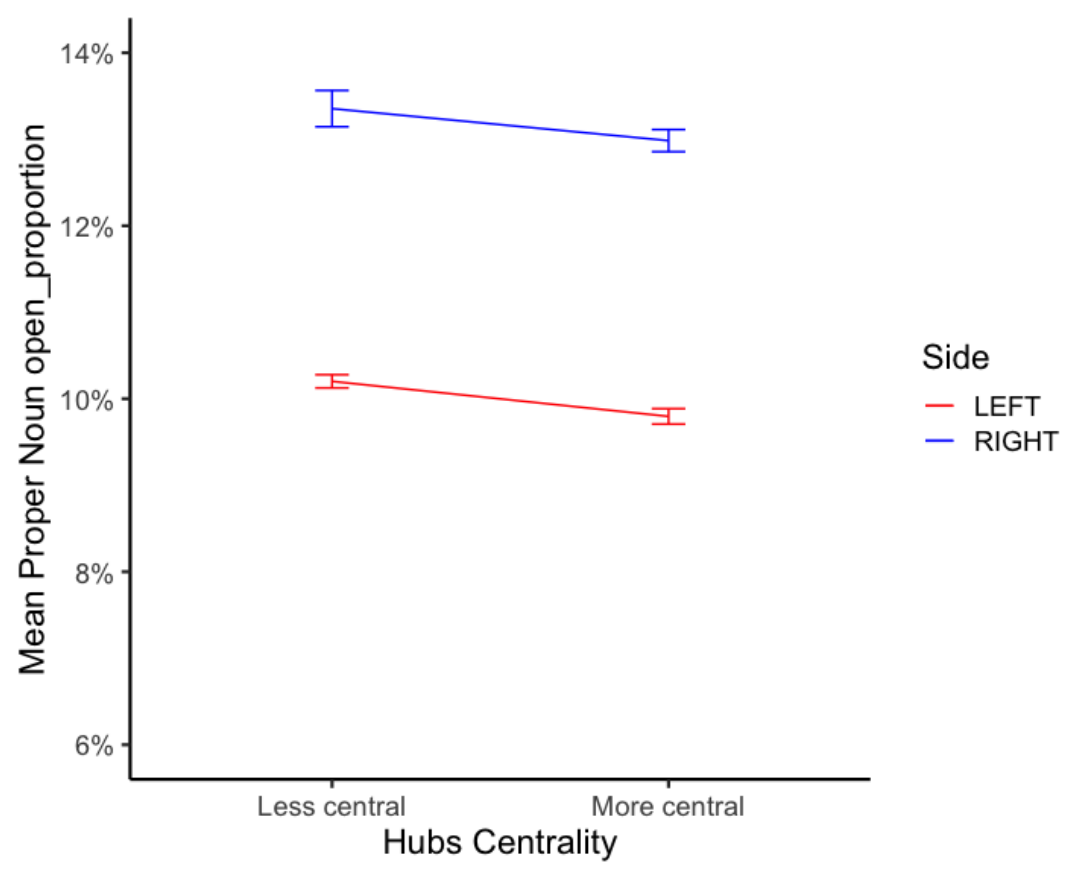

Figure 11. Results of the ANOVA predicting Mean_open_proportion of Proper Nouns per tweet.

\subsection{Mean_proportion_filtered}

The results of a two-way ANOVA, illustrated in Figure 12, indicated a statistically significant main effect of Side $\left(F(1,33954)=242.19, p<1.20 \mathrm{e}-15, \eta_{p}{ }^{2}=0.007\right)$ : on average, Proper Nouns made up a greater mean_proportion_filtered of tweets by RIGHT users than LEFT users. There was no main effect of Centrality $\left(F(1,33954)=2.61, p=.219, \eta_{p}{ }^{2}=0.00008\right)$, and no interaction $\left(F(1,33954)=0.16, p>.999, \eta_{p}{ }^{2}=0.000005\right)$.

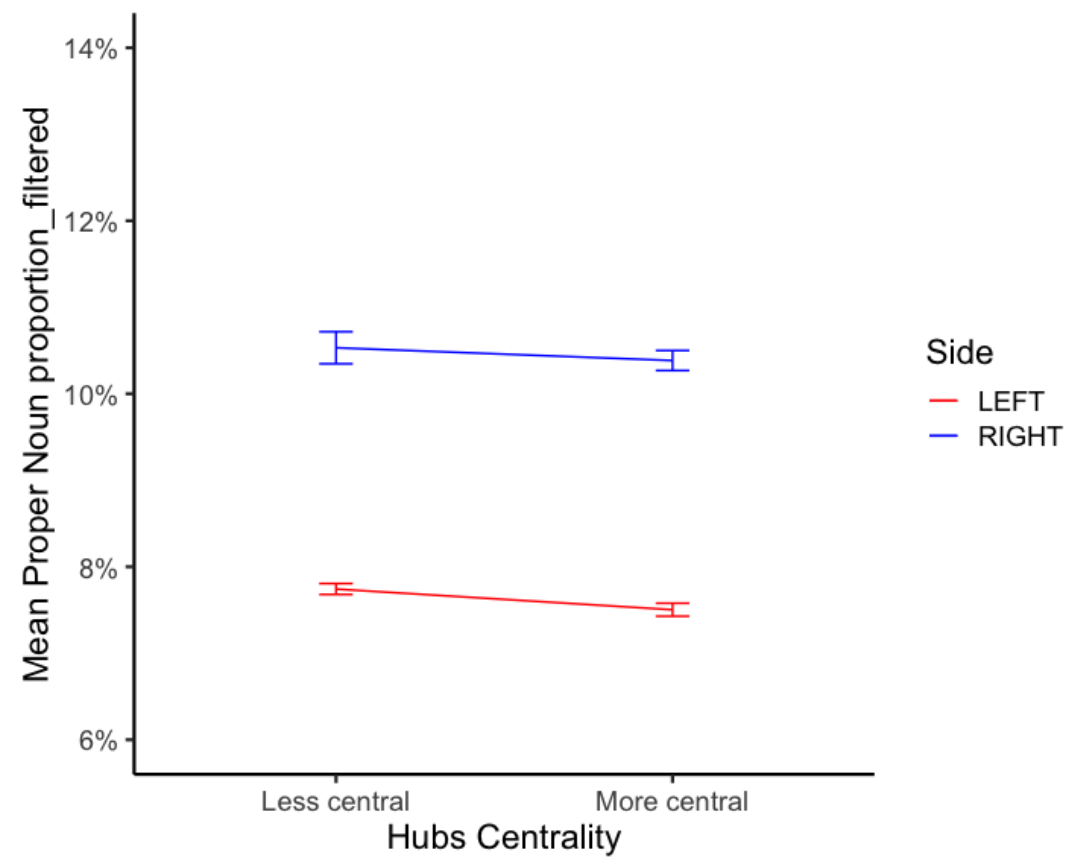

Figure 12. Results of the ANOVA predicting Mean_proportion_filtered of Proper Nouns per tweet. 


\subsection{Mean_proportion}

The results of a two-way ANOVA, illustrated in Figure 13, indicated a statistically significant main effect of Side $\left(F(1,33954)=220.52, p<1.20 \mathrm{e}-15, \eta_{p}{ }^{2}=.006\right)$ : on average, Proper Nouns made up a larger mean_proportion of tweets by RIGHT users than LEFT users. There was no main effect of Centrality $\left(F(1,33954)=2.78, p=.219, \eta_{p}{ }^{2}=0.00009\right)$, and no interaction $\left(F(1,33954)=0.09 p>.999, \eta_{p}{ }^{2}=0.000003\right)$.

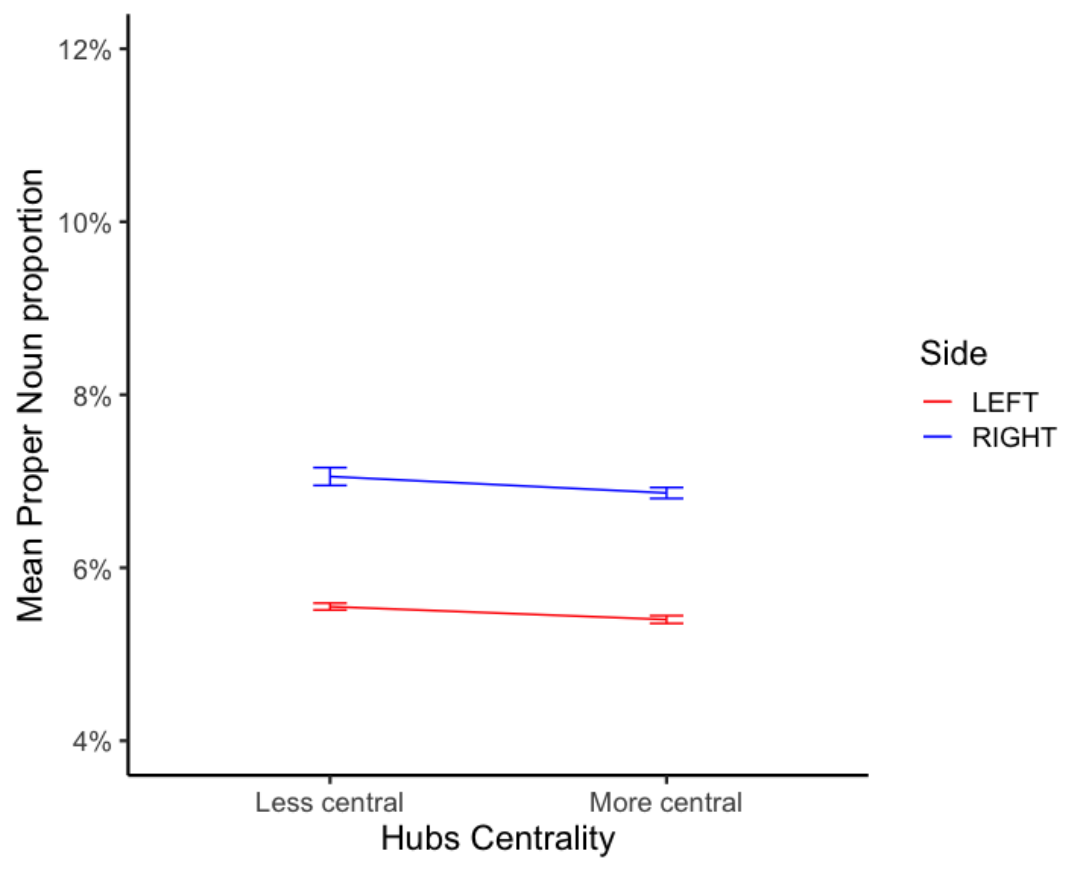

Figure 13. Results of the ANOVA predicting Mean_proportion of Proper Nouns per tweet.

\section{Summary of Results}

\begin{tabular}{|c|c|c|c|}
\hline $\begin{array}{c}\text { Dependent } \\
\text { Variable (DV) }\end{array}$ & Measure of DV & $\begin{array}{c}\text { Effect of Side } \\
\text { (predicted RIGHT } \\
>\text { LEFT) }\end{array}$ & $\begin{array}{l}\text { Effect of Centrality } \\
\text { (predicted More > } \\
\text { Less Central) }\end{array}$ \\
\hline \multirow{7}{*}{$\begin{array}{l}\text { Common } \\
\text { Noun use }\end{array}$} & mean_open_ & - & - \\
\hline & proportion & $* * *$ & $*$ \\
\hline & & $\eta_{p}{ }^{2}=0.05 \%$ & $\eta_{p}{ }^{2}=0.03 \%$ \\
\hline & mean_proportion & & \\
\hline & _ filtered & $\begin{array}{l}\text { Non-sig. } \\
(p=.792)\end{array}$ & $\begin{array}{l}\text { Non-sig. } \\
(p=.112)\end{array}$ \\
\hline & mean_proportion & - & \\
\hline & & $\begin{array}{c}* * * \\
\eta_{p}^{2}=0.2 \%\end{array}$ & $\begin{array}{l}\text { Non-sig. } \\
(p=.385)\end{array}$ \\
\hline
\end{tabular}




\begin{tabular}{|c|c|c|c|}
\hline $\begin{array}{c}\text { Proper Noun } \\
\text { use }\end{array}$ & $\begin{array}{c}\text { mean_open_- } \\
\text { proportion }\end{array}$ & $\begin{array}{c}+ \\
+* * \\
\eta_{p}{ }^{2}=0.7 \%\end{array}$ & $\begin{array}{l}\text { Non-sig. } \\
(p=.080)\end{array}$ \\
\hline & $\begin{array}{c}\text { mean_proportion } \\
\text { _filtered }\end{array}$ & \begin{tabular}{c}
+ \\
\multirow{***}{*}{} \\
$\eta p^{2}=0.7 \%$
\end{tabular} & $\begin{array}{l}\text { Non-sig. } \\
(p=.219)\end{array}$ \\
\hline & mean_proportion & $\begin{array}{c}+ \\
+* * \\
\eta_{p}^{2}=0.6 \%\end{array}$ & $\begin{array}{l}\text { Non-sig. } \\
(p=.219)\end{array}$ \\
\hline
\end{tabular}

Table 4. Summary of ANOVA results predicting Noun use from political Side and network Centrality. '+' represents results as predicted, '- 'results in the opposite direction. '*' indicates $p<. .05$, '**' $p<.01$, ‘***' $p<.001$. No interaction effects were found.

\section{Discussion}

This study set out to test the hypotheses that politically Conservative (here: RIGHT) individuals, and especially individuals more strongly involved in the Conservative networks on Twitter (here: More Central RIGHT users), use more Nouns in spontaneous Twitter communication. Because of the part-of-speech tagging tool used, this study separated Common Nouns from Proper Nouns, and created three different versions of Noun proportion scores for each type of Noun. The patterns of results differed strongly between Common and Proper Nouns, and differed slightly between the different versions of the Noun proportions.

The Twitter data did not support the original hypothesis regarding Common Nouns. In fact, the opposite effect was found - Liberal individuals used more Common Nouns per tweet than Conservative individuals. However, the effect sizes reported $\left(\eta_{p}^{2}=0.05 \%\right.$ to $\left.0.2 \%\right)$ were nowhere near comparable to that of Cichocka et al.'s Study 3 (Cohen's $d=0.50$ ). Furthermore, the effect for Common Nouns was not consistent across all three proportion scores. Thus, the reversal in the direction of the effect for Common Nouns may simply be a chance finding due to the large sample size.

The effect for Proper Nouns, on the other hand, was in the direction expected for Nouns generally (based on past survey results and the results of Study 1) - the RIGHT used more Proper Nouns proportionally per tweet than the LEFT, and this difference was consistent across three different proportion scores. Effect sizes for this difference were larger than for Common Nouns, but still very small in absolute terms $\left(\eta_{p}^{2}=0.6 \%\right.$ to $\left.0.7 \%\right)$, possibly suggesting that Twitter-based measures of political leaning may be good descriptors but weak predictors of Noun use in practice. 
A key difference between this study and Cichocka et al.'s (2016) Study 3 is our distinction between Common and Proper Nouns, whereas Cichocka et al.'s use of CLARINPL counted both under one group 'Nouns'. Thus, it is possible that the results in Cichocka et al.'s Study 3 were confounded by Proper Nouns. At the same time, however, the mean_open_proportion of Common Nouns per tweet in this study (i.e. the most controlled proportion score) was similar in range to that of Cichocka et al.'s proportions of Nouns per political speech ( $M=29 \%$ on the LEFT and $M=28 \%$ on the RIGHT; $M=0.25$ in Cichocka et al.), so perhaps this study's Common Noun measure is still closer to Cichocka et al.'s Noun proportions than our Proper Noun scores. More generally, the similarity in means suggests that tweets provide a similar domain to that of politicians' speeches for testing the presence of Nouns, with the advantage that they are spontaneous and are available for a large number of voters. Yet, the effect sizes discovered are much smaller - we discuss this subsequently.

This finding could be influenced by linguistic form, linguistic content, or the methods used. In terms of linguistic form, it may be that the same psychological mechanisms that cause Conservatives to use Common Nouns in questionnaires make them use Proper Nouns in spontaneous Twitter communication. These mechanisms could be related to traits Need for Closure (Webster et al., 1997, as suggested by Cichocka et al., 2016) or Essentialism (as suggested in Study 1). Alternatively, it is possible that Conservatives prefer to use more specific labels, perhaps related to their preference for clearer, less complex language (Schoonvelde, Brosius, Schumacher, \& Bakker, 2019). On Twitter, Proper rather than Common Nouns may represent this simplicity and specificity. Alternatively, Conservatives may prefer more formal versions of labels than Liberals, such as full, longer versions of Proper Names (e.g. "Boris Johnson", which would be tagged as two Proper Nouns, instead of "Johnson"). Follow-up analyses showed that, on average, Conservatives in our sample indeed used more pairs of Proper Nouns per tweet $(M=19.5 \%, S D=28.2 \%)$ than Liberals $(M=17.5 \%$, $S D=20.6 \%$; full results reported in Appendix $M$ ).

Assessing the potential influences of linguistic content on these findings, it is possible that Conservatives on Twitter talk more about specific individuals and entities, which happen to be referred to using Proper Nouns (e.g. UK, Trump, God, China, as suggested by the word frequency analyses, see Figure 5). At the same time, Conservatives may generally talk more about objects or entities (which, in the specific context of Twitter, tend to be referred to using Proper Nouns), while Liberals may more frequently talk about actions or qualities (referred to using Verbs and Adjectives). These content-based interpretations could not be addressed in 
this study due to its focus on language form, and on Nouns specifically rather than Adjectives of Verbs.

Finally, the specific methods of this study may have influenced the findings regarding Proper Nouns. Firstly, the results could have been influenced by mistakes in the part-of-speech tagging: some Common Nouns (e.g. "president") were mis-classified as Proper Nouns. The creators of TweetNLP warn that this may result from inconsistencies in the use of capital letters and word order in tweets (Owoputi et al., 2013). Within the 100 most commonly used Proper Nouns (see Appendix E), there were nine mis-classified Common Nouns on the LEFT and eight on the RIGHT, although the judgment as to whether these were mis-classification is itself subject to debate (for example, "park" and "king" may be used as Common Nouns or as Proper Names). Secondly, the proportion scores used may have been influenced by varying content and/or length of tweets. Unlike with the fixed sentence length items in questionnaires, on Twitter the length of tweets can vary from one word to 280. Thus, for example, if Conservatives generally used shorter tweets and talked more about entities, then Proper Nouns would 'weigh' more in their proportion scores. A follow-up analysis to check the average length of tweets on both sides confirmed that the tweets of RIGHT users were indeed, on average, shorter $(M=16.8$ words, $S D=8.3$ ) than the tweets of LEFT users ( $M=19.5$ words, $S D=7.4$; full results reported in Appendix M). That said, this issue of potentially inconsistent sentence length on the Left versus the Right could also have been prevalent in Cichocka et al.'s (2016) analysis of political speeches, although perhaps to a lesser extent due to the formal setting and the likely longer sentences used in official communication. Finally, the mere size of our data set could have caused false positive results (Lin, Lucas, \& Shmueli, 2013), along with the $S D$ values being larger for Proper Noun scores and for RIGHT users.

With all these potential interpretations and caveats in mind, one key difference between this study and linguistic surveys is that, on Twitter, language is used in context. On the social networking site, tweets are often sent out in response to other tweets or breaking news, or as part of a longer discussion. By definition, Proper Nouns refer to specific entities in the world, and Twitter is one of the places where individuals spontaneously discuss specific events, individuals and countries. Perhaps the contrast in the present findings and those from questionnaire studies (Cichocka et al., 2016, Crawford, 2018, and Study 1 above) can be explained by this key difference in the influences of context on grammatical choices.

The effect of Centrality in this study remained somewhat uncertain. Based on the assumption that Hubs Centrality would measure the strength of each user's ideology, and on the findings with continuous measures of ideology from Cichocka et al. and others, this study 
expected to find an interaction of Side and Centrality. Yet, the results showed no such interaction, and largely no effect of Centrality in the main analyses. It is possible that no effects of Centrality were found due to the fact that a very conservative version of the ANOVA was used (Type III sum-of-squares). The robustness checks in the form of five ANOVAs re-run on randomly selected groups of balanced size, reported in Appendix I, suggested a potential effect of Centrality, but in the opposite direction - in 18 out of the 30 models, More Central users were found to use fewer Nouns on average than Less central users. However, these differences were not consistent across all 30 models, and, considering the large size of the sample in this study, these may also be false positives. Reflecting on Centrality more generally, the analysis of some users' profiles and a consideration of past literature (Abramowitz, 2010; PreoţiucPietro et al., 2017) suggested that activity on social networking sites likely demonstrates strength of political engagement rather strength of ideology. There are no studies, to our knowledge, suggesting that more politically engaged users may use Nouns more frequently. This might explain the lack of effects of our Centrality measure on Nouns. Finally, since the Centrality measure did not control for the total amount of (not only political) accounts that a user follows, we might have incorrectly tagged generally more active Twitter users as 'More Central' and thus supposedly having stronger political leanings. This limitation can be addressed in a future design by controlling for the total amount of Twitter 'friends' of each account before calculating network centrality.

In summary, the Twitter data reported here did not support the effect for Common Nouns, contradicting past findings, but revealed a potential effect for Proper Nouns, which might have been masked in the original survey approach (Study 1) or in past analysis of politicians' speeches (Cichocka et al., 2016). Considering this inconsistency and the aforementioned downsides of both Twitter data and questionnaires, a combination of findings from web-based social networks and surveys might prove most informative. This is why this study was followed up with a more controlled questionnaire, which considered Common Nouns and Proper Nouns separately. 


\section{Study 3 - Common Nouns, Proper Nouns and Social Conservatism, with Essentialism and Decisiveness as potential mediators.}

\section{Introduction}

Study 2, using data from the social networking site Twitter, provided an interesting novel suggestion - that perhaps it is Proper Nouns, rather than any Nouns in general, that are preferred by Conservatives. Study 2 did not demonstrate a preference for Common Nouns in Conservatives, contradicting the conclusions form Study 1. This can be interpreted in multiple ways. First, it may be that Noun use on Twitter simply differs from what people say in surveys. Alternatively, the results from Study 2 may have been confounded by other effects (such as linguistic content), or the lack of methodological control, leaving the results open to multiple other interpretations, as discussed above. Finally, these results may indeed point at a more general difference in Proper Noun use between Liberals and Conservatives. If such a general difference exists, then the effect should also show up in a questionnaire setting. To our knowledge, no questionnaire studies exist to date assessing the associations between political attitudes and the use of Proper Nouns. Thus, this study set out to look for such converging evidence, creating novel Proper Noun items in a format similar to Cichocka et al. (2016).

Study 2 was unable to look at Social Conservatism specifically. Common Noun use is associated with the Social facet of political Conservatism (Cichocka et al., 2016; Crawford, 2018; and our Study 1), and, linguistically, Proper Nouns belong to the same broader category of Nouns, so this study hypothesised that Proper Noun use would also be related to Social Conservatism. Similar to Cichocka et al. and Crawford, and expanding on the findings of Study 1, this study distinguished between Implicit and Explicit measures of Social Conservatism, measuring the former as in Study 1 (using the mean of the Right-Wing Authoritarianism and anti-Immigration scales; Fieldhouse et al., 2018), and the latter using a self-placement Likert scale similar to that in Cichocka et al.

Furthermore, this study aimed to further investigate the theoretical suggestions from Studies 1 and 2. One of the interpretations of the Twitter findings was that they may reflect a preference of Conservatives for more definitive language than Liberals, resulting in their use of more specific labels, namely Proper Nouns. To test this explanation, this study re-introduced Need for Closure, or the preference for certainty and an aversion toward ambiguity (Webster \& Kruglanski, 1994), to the discussion. As discussed, past research has distinguished two statistically unrelated traits that may underly Need for Closure (Neuberg et al., 1997) - Personal Need for Structure versus Decisiveness. By definition, Decisiveness is more likely to tap into 
the desire to use language more definitively, and thus allows us to test the 'definitiveness' interpretation of the Twitter findings. It is also worth exploring the role of Decisiveness here to clarify the inconsistencies in past results with Common Nouns - Crawford (2018) found that Decisiveness correlated with both Noun use and Explicit Social Conservatism, whereas in Cichocka et al.'s (2016) sample Decisiveness did not play a role. Furthermore, a new, more specific Decisiveness scale (Roets \& Van Hiel, 2007) could allow for a more focused exploration of Decisiveness in relation to grammar use and Social Conservatism. For these reasons, Decisiveness was chosen as a potential explanatory variable in this study. Theoretically, if Decisiveness mediates the association between Noun use and politics, then the desire to make decisions quickly may explain the tendency of Social Conservatives to use the more definitive part-of-speech Nouns; or vice versa, Decisiveness may lead individuals who naturally prefer Nouns to also develop Social Conservative attitudes.

Finally, Study 1 found that the trait Essentialism (Haslam et al., 2000), and specifically its Discreteness (Bastian \& Haslam, 2006) and Implicit Person Theory facets (IPT, Levy et al., 1998), were associated with both Noun use and Social Conservatism. Discreteness specifically may have acted as a mediator, although this demanded further investigation in larger samples. If Proper Nouns facilitate the psychological trait Essentialism as Common Nouns do (Carnaghi et al., 2008), then Essentialism should also correlate with Proper Noun use. Thus, Essentialism was included in this study as another potential variable of interest. A mediating role of Essentialism, if it was found, could be interpreted as follows: Social Conservatives perceive entities in the world in general, or individuals in particular (relating to Discreteness and IPT especially) in terms of their underlying unchanging essences (e.g. Bastian \& Haslam, 2008), and hence use nominal descriptions, which better satisfy this perception (Carnaghi et al., 2008; Graf et al., 2013); or, alternatively, individuals who naturally prefer Nouns in their speech may also possess stronger trait Essentialism, which causes them to develop more Socially Conservative attitudes. Thus, this study aimed to test Essentialism and Decisiveness against each other as potential mediators of the Noun-conservatism association.

The analysis in this study was separated into two parts. The first part involved a conceptual replication of Cichocka et al. (2016) and Crawford (2018), borrowing the (Common) Noun items from Crawford, as in Study 1, and adding the two potential mediators. The following hypotheses were tested for Common Nouns:

H1. People higher on Implicit Social Conservatism use more (Common) Nouns, as in Cichocka et al. (2016) and Study 1 reported above. 
H2. People higher on Explicit Social Conservatism use more (Common) Nouns, as in Cichocka et al. (2016) and Crawford (2018).

H3. People higher on Discreteness use more (Common) Nouns, as in Study 1.

H4. People higher on IPT use more (Common) Nouns, as in Study 1.

H5. People higher on Decisiveness use more (Common) Nouns, as in Crawford (2018).

The second part of this study aimed to validate Twitter findings regarding Proper Nouns using 20 newly created items, designed in a format similar to Cichocka et. al. (2016) - with a single descriptive sentence (e.g. "Billy went to Eton school. Billy is...”) followed by a Proper Noun versus a non-Proper Noun answer option (e.g. "an Etonian” versus "an Eton alumnus").

In constructing these items, Twitter word frequencies from Study 2 were used as inspiration. About a third of the 30 most frequently used Proper Nouns from Twitter were names (e.g. "Johnson”, see Figure 5). This is in line with the methods used in past research (Carnaghi et al., 2008; Cichocka et al., 2016), which limited the stimuli to descriptions of individuals specifically. Yet, another two-thirds of the 30 most commonly used words on Twitter were descriptions of non-living entities (e.g. "America”, "Sunday"). Considering that the Noun-Conservatism association also appears in descriptions of situations (e.g. "dividing/the division of Jerusalem"; Idan et al., 2018), and in more general speech in Cichocka et al.'s third study, there may be more to Noun use in Conservatives than essentialist or decisive inferences about people's characteristics. To test these contrasting possibilities, we created separate Proper Noun items for descriptions of people and non-living entities (10 items for each). A further analysis of the Twitter word frequencies (see Figure 5) discovered two further sub-groups in each, resulting in four groups of Proper Noun scores: Non-people General (e.g. “China”), Non-people Temporal (e.g. "Friday”), People Characteristics (e.g. “Instagrammer”) and People Names (e.g. “John”). These four scores were used as separate versions of the dependent variable throughout this study. While we tried to keep the format of the questions as close to Cichocka et al.'s stimuli as possible, it was largely not possible to create items that contrasted Proper Nouns against Adjectives or Verbs (as in Cichocka et al., 2016) - this study mainly used Common Noun options as alternatives to the Proper Nouns (e.g. "Sainsbury's” versus “the supermarket”).

The Proper Noun sentences were validated using the same part-of-speech tagger (Owoputi et al., 2013) - only the items with correctly tagged Proper versus Common Nouns were included in our final set of items. The items were also piloted on a sample of ten users and corrected based on qualitative feedback - specifically, we got rid of most political items 
as our pilot participants said their responses to these might have been less intuitive and more ideologically skewed.

While on Twitter Conservatism could not be distinguished into its Implicit-Explicit or Social-Economic facets, the relationships were expected to be similar to those with Common Nouns. Furthermore, the linguistic nature of Proper Nouns may also suggest that these play an 'essentialising' role (Hobbes, 1968), or may facilitate the trait Decisiveness due to their specific, unambiguous nature. Thus, the following hypotheses were tested for Proper Nouns:

H6. People higher on Implicit Social Conservatism use more Proper Nouns, extending the Twitter findings from Study 2.

H7. People higher on Explicit Social Conservatism use more Proper Nouns, extending the Twitter findings from Study 2.

H8. People higher on Discreteness use more Proper Nouns.

H9. People higher on IPT use more Proper Nouns.

H10. People higher on Decisiveness use more Proper Nouns.

If $H 1-5$ or $H 6-10$ above proved to be correct, secondary analyses were planned to see whether IPT, Discreteness or Decisiveness mediate the relationship between (Explicit or Implicit) Social Conservatism and the use of Common Nouns or Proper Nouns. These analyses were pre-registered (pre-registration available at https://aspredicted.org/g22qu.pdf and in Appendix $L$ ). Bearing in mind the demographic influences discovered in Study 1, demographic variables were also considered post-hoc.

\section{Method}

\section{Participants}

Five hundred participants were recruited on Prolific Academic. The sample size was determined in advance, based on the smallest correlation coefficient of $r=.15$ reported by Cichocka et al. (2016), and a power of $\beta=.81$.

Participants were paid $£ 1.37$ for 10 minutes of their time. All participants except the first 20 were pre-screened on Prolific to be British Nationals, born in the UK and have English as their first language. The desired age range was set between 18 and 49 years, aiming to match 70-79\% of the age range of Twitter users (Pew Research Center, 2019a; Statista, 2020b). Participants were recruited on Prolific in batches of 10-50, distributions of Explicit Social Conservatism were checked between these batches for balance, and the batches were released at different times of day. In total, 210 'UK Left' participants and 290 'UK Right' participants 
were requested on Prolific (no 'Centre' users were requested, to match the sampling from Twitter). The data was collected between $31^{\text {st }}$ July 2020 and $2^{\text {nd }}$ August 2020.

A total of five participants were excluded: one did not pass the attention check survey question, one said 'no' on the consent form, one completed the survey in under 3 minutes, and two were not born in the UK or were not native English speakers (these were recruited in the first batch of 20, before the addition of language or country-of-birth pre-screening). Thus, the final sample consisted of 495 participants - 262 female, 232 male (demographics for one participant were not known), aged between 18 and $49(M=30.88, S D=9.05)$.

\section{Procedure}

Participants filled out a questionnaire hosted on Qualtrics Survey Software, consisting of eight sub-questionnaires - Common and Proper Noun use, RWA and anti-Immigration, Explicit Social Conservatism, IPT, Discreteness and Decisiveness. The order of presentation of seven of these was counterbalanced in two groups (survey document available at (https://osf.io/dr7bk/?view_only=27f913c49c7b48019484f784b5db4135) - some users saw the Common and Proper Noun items first, and some last. Within each group, the order of the scales (and, in the case of Proper Nouns, the order of the four categories of questions) was randomised, as were the answer options. After seeing these two sets of measures, participants answered an attention check question and three Explicit Conservatism questions. Demographics were collected automatically from Prolific.

\section{Materials}

\section{Noun scores:}

H1-5 were tested using the 10 Noun items from Crawford (2018). As in Study 1, these were scored as 1 for Noun, and 0 for Adjective/Verb. The resulting score was again a sum of 10 items, referred to as the Noun score throughout the rest of this study, for consistency with Study 1. Descriptive statistics for this and the other variables are available in Table 5 below.

H6-10 were tested using 20 novel Proper Noun items. These were similar in format to Cichocka et al.'s (2016) original items (e.g. “Billy went to Eton school. Finish the sentence 'Billy is ... an Etonian [Proper Noun]/an Eton alumnus [Common Noun].'”). While the majority of non-Proper-Noun options were Common Nouns, there was one item where the alternative was an Adjective ("an American” vs. "American”). 
As described, four groups of Proper Noun scores were created: Non-people General (e.g. "You have decided to visit your favourite cafe, Pret. Finish the sentence - 'On my way to ... Pret/the cafe."”); Non-people Temporal (e.g. "Tomorrow is Friday. Finish the sentence 'I will need to do this ... on Friday/tomorrow."”); People Characteristics (e.g. "Nicola has a popular video blog on the website TikTok. Finish the sentence - Nicola is ... a TikToker/a TikTok star."'); and People Names (e.g. "Your family is visiting your neighbour family - the Stephensons. Finish the sentence - 'On our way to visit ... the Stephensons/the neighbours. '”). The full list of items is presented in Appendix B. Each of these four groups contained five items, with answer options scored as 0 (non-Proper-Noun) or 1 (Proper Noun). The resulting four scores were calculated as sums of the respective five items.

\section{Implicit Social Conservatism:}

To measure Implicit Social Conservatism, as in Study 1, this study used the five-item Right-Wing Authoritarianism (RWA) scale and the four-item anti-Immigration scale from the BES (Fieldhouse et al., 2018). These were scored in the same way as before, with the necessary reverse scoring such that higher scores on both indicated greater Social Conservatism (see Study 1 for details, and Table 5 for descriptive statistics).

The combination of the RWA and anti-Immigration items again showed good internal consistency (Cronbach's alpha=0.89). Thus, the z-scored versions of the two scales were again combined into a single mean score, on which higher values indicated greater Implicit Social Conservatism.

\section{Explicit Social Conservatism:}

To measure Explicit Social Conservatism, a single item was used ("Where on the following scale of political orientation would you place yourself in terms of SOCIAL and CULTURAL issues in particular?"), with answers on a 7-point Likert scale (from 1=Definitely Left to 7=Definitely Right). To keep our survey brief, this scale included fewer answer options than the 11-point Likert scale from Cichocka et al. (2016). Single Overall and Economic items were also included for balance, but were not analysed.

\section{Essentialism:}

Following Study 1, Essentialism was measured using the same Likert scale items from the Discreteness (Bastian \& Haslam, 2006) and Implicit Person Theory scales (IPT; Levy et 
al., 2001). The Discreteness score was again an average of eight items, and the IPT score was an average of three, with higher scores indicating greater Essentialism on both.

As in Study 1, a combination of these two scales did not show good internal consistency (Cronbach's alpha=.62), so these items were kept separate.

\section{Decisiveness:}

We used the updated version of the Decisiveness scale (Roets \& Van Hiel, 2007), consisting of six sentences (e.g. “When I have made a decision, I feel relieved.”). Participants responded on a 6-point Likert scale (from 1=strongly disagree to 6=strongly agree) - the full items are included in Appendix B. The final score was an average of the six items.

\begin{tabular}{|c|c|c|c|c|c|c|c|}
\hline Measure & Items & $M$ & $S D$ & Median & Range & Skew & Kurtosis \\
\hline $\begin{array}{l}\text { Common } \\
\text { Noun score }\end{array}$ & 10 sentences & 4.09 & 1.93 & 4 & $0-10$ & 0.34 & -0.16 \\
\hline \multirow[t]{4}{*}{$\begin{array}{l}\text { Proper Noun } \\
\text { score }\end{array}$} & $\begin{array}{l}\text { Non-people General } \\
-5 \text { sentences }\end{array}$ & 3.48 & 1.00 & 4 & $0-5$ & -0.55 & 0.34 \\
\hline & $\begin{array}{c}\text { Non-people Temporal } \\
-5 \text { sentences }\end{array}$ & 1.20 & 1.10 & 1 & $0-5$ & 1.23 & 1.75 \\
\hline & $\begin{array}{c}\text { People Characteristics } \\
-5 \text { sentences }\end{array}$ & 2.22 & 1.05 & 2 & $0-5$ & -0.07 & 0.49 \\
\hline & $\begin{array}{l}\text { People Names } \\
-5 \text { sentences }\end{array}$ & 2.83 & 1.15 & 3 & $0-5$ & 0.02 & -0.44 \\
\hline $\begin{array}{l}\text { Implicit } \\
\text { Social }\end{array}$ & $R W A-5$ Likert scales & 3.14 & 0.90 & 3.2 & $1-5$ & -0.20 & -0.57 \\
\hline Conservatism & $\begin{array}{c}\text { Immigration }-4 \\
\text { Likert scales, reverse- } \\
\text { scored and } z \text {-scored }\end{array}$ & 0 & 0.91 & -0.12 & $\begin{array}{l}-1.59- \\
1.78\end{array}$ & 0.25 & -0.77 \\
\hline $\begin{array}{c}\text { Explicit } \\
\text { Social } \\
\text { Conservatism } \\
\end{array}$ & 1 Likert scale & 3.75 & 1.92 & 4 & $1-7$ & 0.09 & -1.31 \\
\hline $\begin{array}{c}\text { Discreteness } \\
\text { (Essentialism) }\end{array}$ & 8 Likert scales & 3.24 & 0.42 & 3.25 & $\begin{array}{l}1.12- \\
4.75\end{array}$ & -0.12 & 1.60 \\
\hline $\begin{array}{c}I P T \\
\text { (Essentialism) }\end{array}$ & 3 Likert scales & 3.35 & 0.43 & 3.38 & $2-5$ & 0.06 & 0.27 \\
\hline Decisiveness & 6 Likert scales & 3.84 & 0.84 & 3.83 & $1-6$ & -0.12 & -0.14 \\
\hline
\end{tabular}




\section{Design}

To test $H 1-5$, pairwise correlations were computed with Noun score as the outcome, and Implicit Social Conservatism, Explicit Social Conservatism, IPT, Discreteness and Decisiveness as predictors. Structural equation modelling was then used to test for mediations.

To test H6-10, pairwise correlations were computed between each of the four Proper Noun scores as outcome and the same predictors as above, applying Holm-Bonferroni correction for multiple comparisons.

\section{Results}

A visual inspection of the data suggested a potentially bimodal distribution of Explicit Social Conservatism scores $($ Kurtosis $=-1.31$ ), perhaps due to our manual exclusion of 'Centre' users in Prolific pre-screening. However, past simulations suggest that, in samples of $N>160$, the bias in the correlation coefficient caused by a bimodal distribution is almost zero (Bishara $\&$ Hittner, 2015). Thus, Pearson's $r$ was used in correlation analysis, but the patterns of results were identical for the non-parametric Kendall's tau.

\section{Analyses with Common Nouns}

Testing H1-5, a series of pairwise correlation analyses was run. Noun scores positively correlated with Implicit Social Conservatism $(r=0.126, p=.004)$, confirming $H 1$ and replicating the findings from Cichocka et al. (2016), although with a smaller effect size. Noun use positively correlated with IPT ( $r=0.117, p=.009)$, confirming $H 4$ but with a smaller effect size than in Study 1. Correlation plots and distributions of these variables significantly are presented in Appendix $C$. Noun use was not associated with Explicit Social Conservatism $(p=.621)$, Discreteness ( $p=.099$ ) or Decisiveness ( $p=.296$ ), not supporting $H 2, H 3$ and $H 5$ respectively.

Based on the discovered correlations, a mediation analysis for IPT was performed, using the $R$ package lavaan. The model was significant overall ( $b=.336, S E=.116, p=.004)$. As summarised in Figure 14, the regression of Social Conservatism on IPT was positive and statistically significant $(b=0.225, S E=0.024, p<.001)$. The direct effect of IPT on Noun sum in this model was no longer significant $(b=0.353, S E=0.216, p=.101)$. The path from Social Conservatism to Noun use remained significant after the inclusion of IPT in the model $(b=0.257, S E=0.125, p=.040)$. Thus, IPT did not fully mediate the Noun-Conservatism association. 


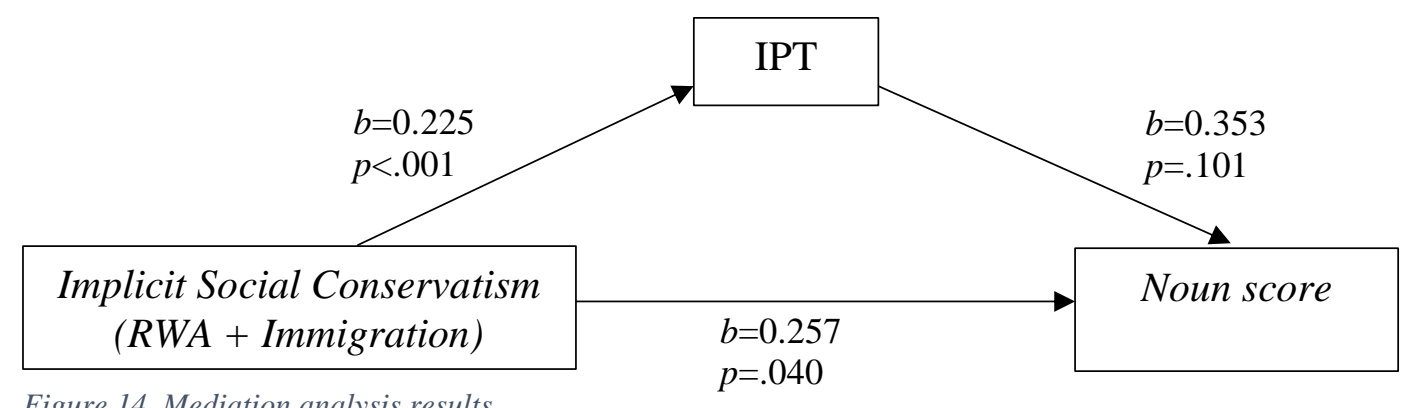

Figure 14. Mediation analysis results.

\section{Analyses with Proper Nouns}

Testing H6-10 for each of the four Proper Noun scores, four correlation analyses we performed using Holm-Bonferroni correction for each of the five hypotheses. As shown in Table 6, none of the five hypotheses were supported. Uncorrected $p$-values suggested the same lack of effect. No mediation analyses were therefore performed.

\begin{tabular}{llllll}
$\begin{array}{l}\text { Outcome } \\
\text { Variable } \\
\text { (Proper Noun } \\
\text { score })\end{array}$ & $\begin{array}{l}\text { Implicit } \\
\text { Social } \\
\text { Conservatism } \\
(\text { H6) }\end{array}$ & $\begin{array}{l}\text { Explicit } \\
\text { Social } \\
\text { Conservatism } \\
(\text { H7) }\end{array}$ & $\begin{array}{l}\text { Discreteness } \\
(\text { H8) }\end{array}$ & IPT $($ H9) & $\begin{array}{l}\text { Decisiveness } \\
(\text { H10 })\end{array}$ \\
\hline $\begin{array}{l}\text { Non-people } \\
\text { General }\end{array}$ & $p=.615$ & $p>.999$ & $p>.999$ & $p>.999$ & $p=.692$ \\
$\begin{array}{l}\text { Non-people } \\
\text { Temporal }\end{array}$ & $p=.776$ & $p>.999$ & $p>.999$ & $p>.999$ & $p=.692$ \\
$\begin{array}{l}\text { People } \\
\text { Characteristics }\end{array}$ & $p=.304$ & $p=.744$ & $p=.938$ & $p>.999$ & $p>.999$ \\
$\begin{array}{l}\text { People Names } \\
p=.776\end{array}$ & $p>.999$ & $p>.999$ & $p>.999$ & $p>.999$ \\
\hline
\end{tabular}

Table 6. Pairwise correlations between the four Proper Noun scores and the other predictor variables (with the relevant hypotheses listed in brackets), with Holm-Bonferroni-corrected p-values.

\section{Post-hoc analyses}

Considering the differing patterns of results obtained with Implicit versus Explicit Social Conservatism measures (only the former correlated with Noun use), as well as the differences in their mean values (see Table 5), it is possible that these two measures were not picking out the same identifying characteristic. Nevertheless, a correlation analysis found that Implicit and Explicit Social Conservatism scores were highly correlated ( $r=.654, p=2.2 \mathrm{e}-16)$.

Following on from Study 1, we considered the influence of demographic variables. The mean scores of Common Noun use differed marginally by sex $(t(488)=-2.0306, p=.043$, Cohen's $d=0.182)$, with female participants using fewer Common Nouns $(M=3.9, S D=1.91)$ 
than male participants $(M=4.3, S D=1.93)$. Age did not correlate with Common Noun use $(p=.097)$. A generalised linear model predicting Common Noun scores from Implicit Social Conservatism, sex and IPT was overall significant $(p=4.8 \mathrm{e}-04)$, but only IPT remained a significant predictor $(b=0.240, p=.015)$.

For Proper Nouns, we only found an association between Temporal Proper Noun scores and sex $(t(435)=-2.812, p=.021$, Cohen's $d=0.256)$, with female participants using fewer Temporal Proper Nouns on average $(M=1.06, S D=0.94)$ than male users $(M=1.34, S D=1.22)$. Age did not correlate with any of the Proper Noun scores.

\section{Discussion}

Study 3 set out to test and further clarify the findings from Cichocka et al. (2016), Crawford (2018) and Studies 1 and 2 reported here. Common Noun scores only correlated with Implicit Social Conservatism and IPT, overlapping with the findings from Study 1, but not with Explicit Social Conservatism (contradicting Cichocka et al.'s study 1 and Crawford), Discreteness (contradicting Study 1 here) or Decisiveness. Follow-up mediation analyses did not support the role of IPT as a mediator of the Noun-Conservatism association. Finally, after controlling for both IPT and sex, Implicit Social Conservatism was no longer a significant predictor of Common Noun use.

The results with Proper Nouns, using newly constructed questionnaire items, did not confirm the Twitter findings from Study 2 - there was no evidence of Social Conservatives using more Proper Nouns in their answers on any of the four groups of items, for either Implicit or Explicit Conservatism measures. Further, there was no evidence for an effect of the other predictors of interest - IPT, Discreteness or Decisiveness - on Proper Noun use, contradicting our hypotheses.

Reflecting on these findings, we must briefly discuss the discrepancies between our Implicit and Explicit Conservatism measures. These were intended to measure the same underlying concept - Social Conservatism. Yet, the correlation between these two measures, while strong, was not complete $(r=.654)$. Furthermore, only the Implicit measure was associated with Noun use - this mirrors the findings of Cichocka et al. (2016), but goes against the results of Crawford (2018). In theory, the finding for the Implicit measure suggests that the influence of politics on Noun use, should it exist at all, might be sub-conscious, in which case the Implicit measure is likely a more appropriate classifier of political attitude in this study. However, as discussed, the Implicit scale does not tap into all aspects of conservative ideology, 
and both scales may suffer from social desirability bias, whereby participants may bias their responses towards what they believe may be the more socially desirable ones (Krumpal, 2013). This is why we developed the new measure in Study 2.

It is possible that this study missed an association between Decisiveness and Common Noun use, found by Crawford (2018), due to the newer Decisiveness measure used here. Roets and Van Hiel (2007) created this updated scale to distinguish two underlying facets of Decisiveness. They argue that the old measure (Webster \& Kruglanski, 1994), which was used by Crawford and Cichocka et al. (2016), was confounding the ability to make quick decisions with the motivation or need to do so (Mannetti et al., 2002). Perhaps is it this 'ability' facet of Decisiveness, dropped from the new scale, that made it associate with Noun use in previous studies (Cichocka et al., 2016; Crawford, 2018). Alternatively, the present results, and the lack of an association with Decisiveness in Cichocka et al., may simply suggest that the 'definitiveness' explanation of Noun use in Conservatives is not valid.

The lack of effects for Proper Nouns in this study can be interpreted in multiple ways. First, the Twitter findings could have been a false positive resulting from a large sample, thus not replicating in the survey data reported here. However, Proper Noun scores in this study did not correlate with Essentialism or Decisiveness either, although this association was expected based on linguistic theory (Hobbes, 1968). This may point to more general problems with our Proper Noun measure.

Second, the lack of effect for Proper Nouns in this study may shed light onto another content-specific feature of Proper Nouns, in addition to those discussed in Study 2. In this study, the Proper Noun items did not describe political figures, beyond the two items about the prime minister and the leader of the Labour party (see Appendix B). Yet, the word frequency analyses in Study 2 (see Figure 5 for the top 30 words on each side, and Appendix E for the top 100) suggested that many of the frequently used Proper Nouns were potentially politically loaded terms - for example, the name "Biden”, referring to a Left-wing politician, was used more frequently on the Right. It may thus be the case that Conservative users used more Proper Nouns than Liberals on Twitter because they were more likely to talk about politics, and perhaps about political entities with opposing views. To test this, a follow-up questionnaire could create political Proper Noun items and re-test their use by Conservative versus Liberal participants.

Finally, while efforts were made to create sentence completion items that would function in the same way as the Noun items from Cichocka et al. (2016), it is not easy to validate findings from spontaneous speech on Twitter in a controlled questionnaire setting. On 
Twitter, people's conversations span over numerous tweets and multiple days, are filled with hashtags or mentions, and are embedded in a larger context of breaking news or current events happening in the world. Such context is impossible to replicate in a questionnaire. Yet, in the presence of such context, the use of Proper Nouns likely becomes common, as people talk about specific entities and individuals. Confirming this suggestion, our word counts data from Study 2 (see Figure 5) suggest that voters on Twitter frequently discuss both current political leaders (e.g. "Boris Johnson”) and frequently discussed entities such as countries (e.g. “China”). In a questionnaire study with Noun items similar to Cichocka et al. (2016), individuals do not have such event-specific, temporal or conversational context, they are not engaged in conversation with other participants, and they are not immediately exposed to outside information. Thus, the way participants end up choosing to use Nouns in a controlled, decontextualised questionnaire setting may be very different from the way they choose to communicate on Twitter. Confirming this, some of the participants in this study commented that the Proper Noun items were difficult to respond to without context, saying they would have preferred to choose 'neither' instead of either of the options. This lack of context may have prevented this study from replicating the association between political leaning and Proper Nouns, should such an association actually exist. 


\section{General Discussion}

This three-part study, using a mixture of survey and web-based methodologies, provided some UK-based evidence for an association between political Conservatism and Noun use, adding to the results reported by Cichocka et al. (2016) and Crawford (2018). It also offered novel insights into the finer details of the effect, potential underlying mechanisms and possible confounding demographic variables.

Study 1 replicated Cichocka et al.'s (2016) finding in a UK sample, using a Right-Wing Authoritarianism (RWA) scale and an anti-Immigration scale as the somewhat less countryspecific measures of Implicit Social Conservatism. The size of correlations obtained between Noun use and Conservatism was similar to that of Cichocka et al. ( $r=.15$ in both). Demographic analyses suggested a potential confounding role of education, but regression analysis controlling for this suggested that the trait Essentialism, and specifically its Discreteness aspect (Bastian \& Haslam, 2006), might have a stronger influence on Noun use than politics or education.

Study 2 assessed this effect in spontaneous communication on Twitter. It distinguished between Common Nouns and Proper Nouns, created three different Noun proportion scores and used network-based measures of political attitude. Using a categorical measure of political Side, Study 2 found that Conservatives used more Proper Nouns per tweet than Liberals, with effect sizes of $\eta_{p}^{2}=0.6 \%$ to $0.7 \%$. The effect with Common Nouns was smaller and less consistent, but pointed in the opposite direction, with Conservatives using fewer Common Nouns per tweet. This Twitter study was unable to consider Social Conservatism specifically, or to explore the role of traits such as Essentialism. A network Centrality measure was also used to represent the strength of one's political ideology; however, validation analyses showed that this measure likely pointed towards political engagement instead. Main results with this measure were generally not significant, but subsequent ANOVAs run as robustness checks on smaller samples suggested that more Central users may use fewer Nouns per tweet.

Study 3 aimed to explore the newly discovered effects for Proper Nouns in a more controlled questionnaire setting, but did not confirm the Twitter correlation between Conservatism and Noun use. Study 3 also re-tested the results from Cichocka et al. (2016) and Study 1, but including both Implicit and Explicit measures of Social Conservatism. It only replicated the association between Implicit Social Conservatism and Common Noun use. Seeking to further refine the mediation hypotheses in light of the findings from Studies 1 and 2, Study 3 also contrasted the roles of Decisiveness versus Essentialism, and accounted for the 
potential confounds age and sex. The results did not provide evidence for either Decisiveness or Essentialism as potential mediators of the Noun-Conservatism association, but suggested the stronger influence of the IPT facet of Essentialism over sex or politics.

The demographic analyses reported in these three studies suggest that past correlations between Conservatism and Noun use (Cichocka et al., 2016; Crawford, 2018) may have been confounded by the negative correlations with education level, or a stronger preference for Nouns (i.e. both Common and Proper) in males. However, Studies 1 and 3 did not provide converging evidence of the influence of either of these on Noun use. This highlights the importance of including these variables in follow-up studies to improve our understanding.

\section{Implications for Theory}

The two survey-based studies reported here appear to demonstrate a preference for Common Nouns in Social Conservatives, but only on Implicit measures. This suggests that fundamental behaviours such as Nouns use in non-political communication can provide a window onto the sub-conscious patterns of thought associated with political ideology.

However, this effect for Common Nouns was not observed in the much larger study of spontaneous speech on Twitter. Furthermore, the survey studies found potential evidence of a confounding role of education level and sex - this, if supported in future studies, would imply that political ideology itself does not necessarily cause or result from a preference for Nouns. Finally, our survey findings suggest that it may be the essentialist thinking about individuals that underlies the Noun-Conservatism association, although the details of this remain uncertain. We found some evidence of a mediating role of Discreteness (i.e. one's belief in the discreteness of existing groups; Bastian \& Haslam, 2006), which makes sense considering the grouping' property of Nouns (Carnaghi et al., 2008; Graf et al., 2013), but this was not supported in Study 3, whereas a correlation between Noun use and IPT (i.e. the belief in the unchanging nature of people's characteristics; Levy et al., 2001, 1998) was discovered in Study 3 but not in Study 1. Thus, by examining the previously reported Noun-Conservatism association and investigating related characteristics, this collection of studies offers the possibility that politics itself may only be related to Noun use through other underlying variables, with Essentialism likely having the strongest influence. Nevertheless, further experimental evidence would be required to investigate any causal pathways.

The null findings for the trait Decisiveness, in theory, reject the proposition that Nouns (Cichocka et al., 2016), including Proper Nouns on Twitter, may be used more by Conservatives due to their disambiguating or definitive nature, although further investigation 
is needed with older measures of Decisiveness. The effects of Personal Need for Structure (Neuberg et al., 1997), suggested as a potential mediator by Cichocka et al., were not investigated here.

This report provides an interesting novel investigation into the use of Proper Nouns by different sides of the political spectrum. This sub-group of Nouns likely would not have been considered if this study hadn't used the contextualised measure of spontaneous language use on Twitter and the Twitter-specific part-of-speech tagger. Our findings suggest that, in spontaneous communication on Twitter, it may be Proper rather than Common Nouns that are preferred by Conservatives. One unresolved issue is that on Twitter the Social aspects of Conservatism could not be distinguished from Economic or Overall Conservative attitudes. Thus, Economic Conservatism, unrelated to Common Nouns in the original study (Cichocka et al., 2016), could have confounded the reported Twitter results, leading to a smaller effect size for Proper Nouns or concealing the effects for Common Nouns. Alternatively, the use of Proper Nouns on Twitter may have been associated with Economic rather than Social Conservatism, explaining the lack of association with Social Conservatism in Study 3.

The greater use of Proper Nouns in Conservatives was not replicated in a decontextualised questionnaire setting (Study 3). Yet, the effect for Common Nouns (Cichocka et al., 2016; Crawford, 2018) did not replicate and potentially went in the opposite direction on Twitter. This discrepancy may be caused by one key difference between the two domains: the presence of context. As discussed above, spontaneous language use, a context-dependent behaviour, may differ from linguistic behaviour in questionnaires, which use short, decontextualised items. This demonstrates a significant disadvantage of survey methods against using real-world speech. Yet, the disadvantages of using web-based observational data such as tweets are also ample, and include biases, errors in language models (e.g. part-ofspeech taggers) and many potential interpretations of any effects found (due to lack of methodological control present in questionnaires). This illustrates the strength of using multiple domains and methods to obtain converging evidence about everyday yet complex behaviours such as language use. Here, a combination of survey and web-based methods allowed for a refinement of both theory and conclusions, demonstrating that real-world associations between Noun use and political leaning may not be as clear as past questionnaire studies suggest.

Finally, we must discuss the small effect sizes reported in this study. Even in the large Twitter sample, the size of the correlation between Noun use and Conservatism remained miniscule. Based on this, the reader should not expect Conservatives to consistently use more Nouns than Liberals. Neither should one predict someone's political orientation from small 
samples of their language use. It is more likely that this effect, should it exist at all, would manifest itself in subtle differences, only detectable in large datasets. The aim of this study was not to create a predictive tool, but to use language as a window into political cognition. Our findings suggest that Noun use is not only such a window, but also may be a result and/or cause of other psychological and demographic differences between individuals (although, yet again, our correlational methods do not allow for causal inferences).

\section{Suggestions for Future Research}

To address some of the methodological challenges of the Twitter study, a future investigation could employ a weighting on the user network based on the Social Conservatism of elites. Such data exists for at least 99 UK MPs (Bale et al., 2020). This weighting would allow for a focus on Social rather than Economic or Overall Conservatism, preventing confounded results and more accurately mirroring existing survey research (Cichocka et al., 2016; Crawford, 2018). Additionally, a fuller network of Twitter users could be collected, including non-political ties like following patterns between voters (e.g. Halberstam \& Knight, 2016), although the political inferences made in this way would require validation through methods such as geographical (Halberstam \& Knight) or voter registration data matching (Barberá, 2015). To represent the network of Twitter interactions even more comprehensively, a multi-mode network could be considered, incorporating not only 'following' activity, but also 're-tweets' and 'mentions' (Gu et al., 2016; Kwak, Lee, Park, \& Moon, 2010); however, the greater influences of activity bias on within-tweet interactions must be noted. Finally, it may be worthwhile exploring language use in the 'centrist' Twitter users, i.e. those following both Left and Right elites, which were excluded from this investigation.

To refine the theoretical conclusions made here, future studies could re-investigate the definitive, disambiguating power of Nouns, and its association with Conservative attitudes, by bringing Personal Need for Structure back to the discussion, as well as the original measure of Decisiveness (Neuberg et al., 1997; Webster \& Kruglanski, 1994).

As suggested, the lack of context in the surveys used here may have concealed potential effects for Proper Nouns. Future studies could create longer stories instead of single-sentence items to investigate politics and Nouns contextually in a controlled survey setting. To further investigate the use of Nouns in spontaneous speech while enjoying the advantages of detailed survey measures, another study could match participant responses on psychological and political questionnaires with their Twitter profiles to obtain language samples. Such matching 
has been performed in training Machine Learning models, using self-reported Twitter handles (Preoţiuc-Pietro et al., 2017), but requires great caution regarding user privacy. Finally, to understand whether past results with Common Nouns might have been confounded by Proper Nouns, the political speeches analysed in Cichocka et al.'s Study 3 could be re-analysed to distinguish Proper and Common Nouns. 


\section{Conclusion}

The present collection of studies provides mixed evidence for grammatical differences in the language of Liberals and Conservatives. The two questionnaire studies confirm that Socially Conservative individuals tend to use more Common Nouns in sentence completion tasks, and find that Essentialism, education level and/or sex may mediate this behaviour, although experimental investigations with larger samples are needed. However, the web-based Twitter investigation does not confirm the effect for Common Nouns, but instead finds a novel preference for Proper Nouns in Conservatives; yet, the effect for Proper Nouns is not replicated in a subsequent questionnaire. One interpretation of this discrepancy is the influence of context - Proper Nouns, defined as names of specific individuals and entities in the world, are only used in real-world contexts. Thus, decontextualised, short questionnaire items may prove insufficient for investigating such behaviour. On the other hand, the Common Noun effect may have been specific to short single-sentence questionnaire items, thus not replicating in spontaneous speech on Twitter. It remains uncertain whether such small political differences in Noun use would be manifested in everyday communication offline. More detailed, storybased questionnaires are proposed to test this interpretation.

These results demonstrate that survey-based studies allow for key theoretical contributions and an exploration of potential confounding variables, but may overlook important contextual aspects of linguistic communication. To address this, we show that insightful Twitter data can be collected in large amounts relatively quickly, and create an opensourced collection of code so that other researchers can replicate or modify the analyses. However, this study also demonstrates the limitations of large-scale linguistic and networkbased data, including population biases, errors in linguistic models and ambiguities in interpretation. A combination of web-based social media observations and survey studies may be the best approach for the advancement of Psychological and Political Science. 


\section{Bibliography}

Abramowitz, A. (2010). The disappearing center engaged citizens, polarization, and American democracy / Alan I. Abramowitz. New Haven: New Haven : Yale University Press, c2010.

Agarwal, P., Sastry, N., \& Wood, E. (2019). Tweeting MPs: Digital Engagement between Citizens and Members of Parliament in the UK. Proceedings of the International AAAI Conference on Web and Social Media, 13(01), 26-37. Retrieved from https://www.aaai.org/ojs/index.php/ICWSM/article/view/3359

Altemeyer, R. A. (1981). Right-wing authoritarianism. Winnipeg, Canada: Univ. Manitoba Press.

Bale, T., Cheung, A., Cowley, P., Wager, A., \& Menon, A. (2020). Mind the Values Gap. London.

Barabási, A.-L., \& Albert, R. (1999). Emergence of Scaling in Random Networks. Science, 286(5439), 509 LP - 512. https://doi.org/10.1126/science.286.5439.509

Barberá, P. (2015). Birds of the Same Feather Tweet Together: Bayesian Ideal Point Estimation Using Twitter Data. Political Analysis, 23(1), 76-91. https://doi.org/10.1093/pan/mpu011

Barberá, P., Jost, J. T., Nagler, J., Tucker, J. A., \& Bonneau, R. (2015). Tweeting From Left to Right: Is Online Political Communication More Than an Echo Chamber? Psychological Science, 26(10), 1531-1542. https://doi.org/10.1177/0956797615594620

Barberá, P., \& Rivero, G. (2014). Understanding the Political Representativeness of Twitter Users. Social Science Computer Review, 33(6), 712-729. https://doi.org/10.1177/0894439314558836

Bastian, B., \& Haslam, N. (2006). Psychological essentialism and stereotype endorsement. Journal of Experimental Social Psychology, 42(2), 228-235. https://doi.org/10.1016/J.JESP.2005.03.003

Bastian, B., \& Haslam, N. (2008). Immigration from the perspective of hosts and immigrants: Roles of psychological essentialism and social identity. Asian Journal of Social Psychology, 11(2), 127-140. https://doi.org/10.1111/j.1467-839X.2008.00250.x

Bishara, A. J., \& Hittner, J. B. (2015). Reducing Bias and Error in the Correlation Coefficient Due to Nonnormality. Educational and Psychological Measurement, 75(5), 785-804. https://doi.org/10.1177/0013164414557639

Boutyline, A., \& Willer, R. (2017). The Social Structure of Political Echo Chambers: Variation in Ideological Homophily in Online Networks. Political Psychology, 38(3), 551-569. https://doi.org/10.1111/pops.12337

Bredart, S., Brennen, T., \& Valentine, T. (2002). The cognitive psychology of proper names. Routledge.

Brin, S., \& Page, L. (1998). The anatomy of a large-scale hypertextual web search engine. Computer Networks, 30, 107-117.

Carnaghi, A., Maass, A., Gresta, S., Bianchi, M., Cadinu, M., \& Arcuri, L. (2008). Nomina Sunt Omina: On the Inductive Potential of Nouns and Adjectives in Person Perception. Journal of Personality and Social Psychology, 94(5), 839-859. https://doi.org/10.1037/0022-3514.94.5.839

Chaney, A. J. B., Stewart, B. M., \& Engelhardt, B. E. (2018). How Algorithmic Confounding in Recommendation Systems Increases Homogeneity and Decreases Utility. In Proceedings of the 12th ACM Conference on Recommender Systems (pp. 224-232). New York, NY, USA: Association for Computing Machinery. https://doi.org/10.1145/3240323.3240370

Cichocka, A., Bilewicz, M., Jost, J. T., Marrouch, N., \& Witkowska, M. (2016). On the 
Grammar of Politics — or Why Conservatives Prefer Nouns. Political Psychology, 37(6), 799-815. https://doi.org/10.1111/pops.12327

Cimpian, A., \& Salomon, E. (2014). The inherence heuristic: An intuitive means of making sense of the world, and a potential precursor to psychological essentialism. Behavioral and Brain Sciences, 37(5), 461.

Colleoni, E., Rozza, A., \& Arvidsson, A. (2014). Echo Chamber or Public Sphere? Predicting Political Orientation and Measuring Political Homophily in Twitter Using Big Data. Journal of Communication, 64(2), 317-332. https://doi.org/10.1111/jcom.12084

Conover, M. D., Gonçalves, B., Flammini, A., \& Menczer, F. (2012). Partisan asymmetries in online political activity. EPJ Data Science, 1(1), 6. https://doi.org/10.1140/epjds6

Conover, M. D., Goncalves, B., Ratkiewicz, J., Flammini, A., \& Menczer, F. (2011). Predicting the Political Alignment of Twitter Users. In 2011 IEEE Third International Conference on Privacy, Security, Risk and Trust and 2011 IEEE Third International Conference on Social Computing (pp. 192-199). https://doi.org/10.1109/PASSAT/SocialCom.2011.34

Conover, M. D., Ratkiewicz, J., Francisco, M., Gonçalves, B., Menczer, F., \& Flammini, A. (2011). Political polarization on twitter. In Fifth international AAAI conference on weblogs and social media.

Conway III, L. G., Houck, S. C., Gornick, L. J., \& Repke, M. A. (2018). Finding the Loch Ness Monster: Left-Wing Authoritarianism in the United States. Political Psychology, 39(5), 1049-1067. https://doi.org/10.1111/pops.12470

Crawford, J. T. (2018). Examining the Effects of Political Orientation on Noun Preference: A Replication of Cichocka et al.(2016). Retrieved from https://doi.org/10.31234/osf.io/m5paz

Duckitt, J., Bizumic, B., Krauss, S. W., \& Heled, E. (2010). A Tripartite Approach to RightWing Authoritarianism: The Authoritarianism-Conservatism-Traditionalism Model. Political Psychology, 31(5), 685-715. https://doi.org/10.1111/j.1467-9221.2010.00781.x

Duckitt, J., Wagner, C., Du Plessis, I., \& Birum, I. (2002). The psychological bases of ideology and prejudice: Testing a dual process model. Journal of Personality and Social Psychology, 83(1), 75.

Eysenck, H. J. (1981). Left-Wing Authoritarianism: Myth or Reality? Political Psychology, 3(1/2), 234-238. https://doi.org/10.2307/3791293

Feinberg, M., \& Willer, R. (2012). The Moral Roots of Environmental Attitudes. Psychological Science, 24(1), 56-62. https://doi.org/10.1177/0956797612449177

Feinberg, M., \& Willer, R. (2019). Moral reframing: A technique for effective and persuasive communication across political divides. Social and Personality Psychology Compass, 13(12), e12501. https://doi.org/10.1111/spc3.12501

Fiedler, K., Semin, G. Ü. N. R., \& Bolten, S. (1989). Language use and reification of social information: Top-down and bottom-up processing in person cognition. European Journal of Social Psychology, 19(4), 271-295. https://doi.org/10.1002/ejsp.2420190403

Fieldhouse, E., Green, J., Evans, G., Schmitt, H., van der Eijk, C., Mellon, J., \& Prosser, C. (2018). British Election Study Combined Wave 1-13 Internet Panel. University of Oxford.

Freeman, J. B., \& Dale, R. (2013). Assessing bimodality to detect the presence of a dual cognitive process. Behavior Research Methods, 45(1), 83-97. https://doi.org/10.3758/s13428-012-0225-x

Gelman, S. A., \& Heyman, G. D. (1999). Carrot-Eaters and Creature-Believers: The Effects of Lexicalization on Children's Inferences About Social Categories. Psychological Science, 10(6), 489-493. https://doi.org/10.1111/1467-9280.00194

Gerber, A. S., Huber, G. A., Doherty, D. D., Dowling, C. M., \& Ha, S. E. (2010). Personality 
and Political Attitudes: Relationships across Issue Domains and Political Contexts. American Political Science Review, 104(01), 111-133. https://doi.org/10.1017/S0003055410000031

González-Bailón, S., Wang, N., Rivero, A., Borge-Holthoefer, J., \& Moreno, Y. (2014). Assessing the bias in samples of large online networks. Social Networks, 38, 16-27. https://doi.org/https://doi.org/10.1016/j.socnet.2014.01.004

Gosling, S. D., Rentfrow, P. J., \& Swann, W. B. (2003). A very brief measure of the Big-Five personality domains. Journal of Research in Personality, 37(6), 504-528. https://doi.org/10.1016/S0092-6566(03)00046-1

Graf, S., Bilewicz, M., Finell, E., \& Geschke, D. (2013). Nouns Cut Slices: Effects of Linguistic Forms on Intergroup Bias. Journal of Language and Social Psychology, 32(1), 62-83. https://doi.org/10.1177/0261927X12463209

Graham, J., Haidt, J., \& Nosek, B. A. (2009). Liberals and Conservatives Rely on Different Sets of Moral Foundations. Journal of Personality and Social Psychology, 96(5), 10291046. https://doi.org/10.1037/a0015141

Gu, Y., Chen, T., Sun, Y., \& Wang, B. (2016). Ideology Detection for Twitter Users with Heterogeneous Types of Links. ArXiv, abs/1612.0. Retrieved from https://arxiv.org/abs/1612.08207

Hagberg, A. A., Schult, D. A., \& Swart, P. J. (2008). Exploring Network Structure, Dynamics, and Function using NetworkX. In G. Varoquaux, T. Vaught, \& J. Millman (Eds.), Proceedings of the 7th Python in Science Conference (pp. 11-15). Pasadena, CA USA.

Halberstam, Y., \& Knight, B. (2016). Homophily, group size, and the diffusion of political information in social networks: Evidence from Twitter. Journal of Public Economics, 143, 73-88. https://doi.org/https://doi.org/10.1016/j.jpubeco.2016.08.011

Hall, D. G. (1991). Acquiring Proper Nouns for Familiar and Unfamiliar Animate Objects: Two-Year-Olds' Word-learning Biases. Child Development, 62(5), 1142-1154. https://doi.org/10.1111/j.1467-8624.1991.tb01595.x

Hargittai, E. (2015). Is bigger always better? Potential biases of big data derived from social network sites. The ANNALS of the American Academy of Political and Social Science, 659(1), 63-76. Retrieved from https://journals.sagepub.com/doi/full/10.1177/0002716215570866

Harnish, R. J., Bridges, K. R., \& Gump, J. T. (2018). Predicting Economic, Social, and Foreign Policy Conservatism: the Role of Right-Wing Authoritarianism, Social Dominance Orientation, Moral Foundations Orientation, and Religious Fundamentalism. Current Psychology, 37(3), 668-679. https://doi.org/10.1007/s12144-016-9552-x

Hartigan, P. M. (1985). Computation of the Dip Statistic to Test for Unimodality. Journal of the Royal Statistical Society. Series C (Applied Statistics), 34(3), 320-325. https://doi.org/10.2307/2347485

Haslam, N., Rothschild, L., \& Ernst, D. (2000). Essentialist beliefs about social categories. British Journal of Social Psychology, 39(1), 113-127. https://doi.org/10.1348/014466600164363

Henrich, J., Heine, S. J., \& Norenzayan, A. (2010). Most people are not WEIRD. Nature, 466(7302), 29. https://doi.org/10.1038/466029a

Hibbing, J. R., Smith, K. B., \& Alford, J. R. (2014). Differences in negativity bias underlie variations in political ideology. Behav Brain Sci, 37(3), 297-307. https://doi.org/10.1017/s0140525x13001192

Himelboim, I., McCreery, S., \& Smith, M. (2013). Birds of a Feather Tweet Together: Integrating Network and Content Analyses to Examine Cross-Ideology Exposure on Twitter. Journal of Computer-Mediated Communication, 18(2), 154-174. 
https://doi.org/10.1111/jcc4.12001

Hobbes, T. (1968). Leviathan. (C. B. Macpherson, Ed.). Harmondsworth: Penguin Books. (Original work published 1651).

Hussak, L. J., \& Cimpian, A. (2018). Investigating the origins of political views: biases in explanation predict conservative attitudes in children and adults. Developmental Science, 21(3), e12567. https://doi.org/10.1111/desc.12567

Idan, O., Halperin, E., Hameiri, B., \& Reifen Tagar, M. (2018). A Rose by Any Other Name? A Subtle Linguistic Cue Impacts Anger and Corresponding Policy Support in Intractable Conflict. Psychological Science, 29(6), 972-983. https://doi.org/10.1177/0956797618772823

Jackson, N., \& Lilleker, D. (2011). Microblogging, Constituency Service and Impression Management: UK MPs and the Use of Twitter. The Journal of Legislative Studies, 17(1), 86-105. https://doi.org/10.1080/13572334.2011.545181

Jost, J. T., Glaser, J., Kruglanski, A. W., \& Sulloway, F. J. (2003). Political conservatism as motivated social cognition. Psychological Bulletin, 129(3), 339.

Kleinberg, J. M. (1999). Authoritative Sources in a Hyperlinked Environment. J. ACM, 46(5), 604-632. https://doi.org/10.1145/324133.324140

Koerner, E. F. K. (1992). The Sapir-Whorf Hypothesis: A Preliminary History and a Bibliographical Essay. Journal of Linguistic Anthropology, 2(2), 173-198. https://doi.org/10.1525/jlin.1992.2.2.173

Krumpal, I. (2013). Determinants of social desirability bias in sensitive surveys: a literature review. Quality \& Quantity, 47(4), 2025-2047. https://doi.org/10.1007/s11135-0119640-9

Kwak, H., Lee, C., Park, H., \& Moon, S. (2010). What is Twitter, a Social Network or a News Media? In Proceedings of the 19th International Conference on World Wide Web (pp. 591-600). New York, NY, USA: Association for Computing Machinery. https://doi.org/10.1145/1772690.1772751

Levy, S. R., Plaks, J. E., Hong, Y., Chiu, C., \& Dweck, C. S. (2001). Static Versus Dynamic Theories and the Perception of Groups: Different Routes to Different Destinations. Personality and Social Psychology Review, 5(2), 156-168. https://doi.org/10.1207/S15327957PSPR0502_6

Levy, S. R., Stroessner, S. J., \& Dweck, C. S. (1998). Stereotype formation and endorsement: The role of implicit theories. Journal of Personality and Social Psychology, 74(6), 1421-1436.

Lin, M., Lucas, H. C., \& Shmueli, G. (2013). Research Commentary-Too Big to Fail: Large Samples and the p-Value Problem. Information Systems Research, 24(4), 906-917. https://doi.org/10.1287/isre.2013.0480

Maass, A. (1999). Linguistic Intergroup Bias: Stereotype Perpetuation Through Language. In M. P. B. T. Zanna (Ed.), Advances in Experimental Social Psychology (Vol. 31, pp. 79121). Academic Press. https://doi.org/https://doi.org/10.1016/S0065-2601(08)60272-5

Mannetti, L., Pierro, A., Kruglanski, A., Taris, T., \& Bezinovic, P. (2002). A cross-cultural study of the Need for Cognitive Closure Scale: Comparing its structure in Croatia, Italy, USA and The Netherlands. British Journal of Social Psychology, 41(1), 139-156. https://doi.org/10.1348/014466602165108

Mavor, K. I., Louis, W. R., \& Sibley, C. G. (2010). A bias-corrected exploratory and confirmatory factor analysis of right-wing authoritarianism: Support for a three-factor structure. Personality and Individual Differences, 48(1), 28-33. https://doi.org/https://doi.org/10.1016/j.paid.2009.08.006

McPherson, M., Smith-Lovin, L., \& Cook, J. M. (2001). Birds of a Feather: Homophily in Social Networks. Annual Review of Sociology, 27(1), 415-444. 
https://doi.org/10.1146/annurev.soc.27.1.415

Mellon, J., \& Prosser, C. (2017). Twitter and Facebook are not representative of the general population: Political attitudes and demographics of British social media users. Research \& Politics, 4(3), 2053168017720008. https://doi.org/10.1177/2053168017720008

Merriam-Webster. (n.d.). Coronavirus. Retrieved August 29, 2020, from https://www.merriam-webster.com/dictionary/coronavirus

Neuberg, S. L., Judice, T. N., \& West, S. G. (1997). What the Need for Closure Scale measures and what it does not: Toward differentiating among related epistemic motives. Journal of Personality and Social Psychology, 72(6), 1396.

Newman, M. E. J. (Mark E. J. . (2010). Networks : an Introduction. Oxford : Oxford University Press, 2010.

Olteanu, A., Castillo, C., Diaz, F., \& Kicıman, E. (2019). Social Data: Biases, Methodological Pitfalls, and Ethical Boundaries. Frontiers in Big Data, 2, 13. Retrieved from https://www.frontiersin.org/article/10.3389/fdata.2019.00013

Owoputi, O., O’Connor, B., Dyer, C., Gimpel, K., Schneider, N., \& Smith, N. A. (2013). Improved part-of-speech tagging for online conversational text with word clusters. In Proceedings of the 2013 conference of the North American chapter of the association for computational linguistics: human language technologies (pp. 380-390).

Pew Research Center. (2019a). National Politics on Twitter: Small Share of U.S. Adults Produce Majority of Tweets.

Pew Research Center. (2019b). Sizing Up Twitter Users.

Pfister, R., Schwarz, K., Janczyk, M., Dale, R., \& Freeman, J. (2013). Good things peak in pairs: a note on the bimodality coefficient. Frontiers in Psychology. Retrieved from https://www.frontiersin.org/article/10.3389/fpsyg.2013.00700

Preoţiuc-Pietro, D., Liu, Y., Hopkins, D., \& Ungar, L. (2017). Beyond binary labels: political ideology prediction of twitter users. In Proceedings of the 55th Annual Meeting of the Association for Computational Linguistics (Volume 1: Long Papers) (pp. 729-740). Retrieved from https://www.aclweb.org/anthology/P17-1068.pdf

Rangel, U., \& Keller, J. (2011). Essentialism Goes Social: Belief in Social Determinism as a Component of Psychological Essentialism. Journal of Personality and Social Psychology, 100(6), 1056-1078. https://doi.org/10.1037/a0022401

Robson, J., Marshall, J., Pring, T., Montagu, A., \& Chiat, S. (2004). Processing proper nouns in aphasia: Evidence from assessment and therapy. Aphasiology, 18(10), 917-935. https://doi.org/10.1080/02687030444000462

Roets, A., \& Van Hiel, A. (2007). Separating Ability From Need: Clarifying the Dimensional Structure of the Need for Closure Scale. Personality and Social Psychology Bulletin, 33(2), 266-280. https://doi.org/10.1177/0146167206294744

Šćepanović, S., Mishkovski, I., Gonçalves, B., Nguyen, T. H., \& Hui, P. (2017). Semantic homophily in online communication: Evidence from Twitter. Online Social Networks and Media, 2, 1-18. https://doi.org/https://doi.org/10.1016/j.osnem.2017.06.001

Schoonvelde, M., Brosius, A., Schumacher, G., \& Bakker, B. N. (2019). Liberals lecture, conservatives communicate: Analyzing complexity and ideology in 381,609 political speeches. PLOS ONE, 14(2), e0208450. Retrieved from https://doi.org/10.1371/journal.pone.0208450

Semin, G. R., \& Fiedler, K. (1991). The Linguistic Category Model, its Bases, Applications and Range. European Review of Social Psychology, 2(1), 1-30. https://doi.org/10.1080/14792779143000006

Simmons, J. P., Nelson, L. D., \& Simonsohn, U. (2012). A 21 Word Solution. Retrieved August 3, 2020, from http://dx.doi.org/10.2139/ssrn.2160588

Slatcher, R. B., Chung, C. K., Pennebaker, J. W., \& Stone, L. D. (2007). Winning words: 
Individual differences in linguistic style among U.S. presidential and vice presidential candidates. Journal of Research in Personality, 41(1), 63-75.

https://doi.org/https://doi.org/10.1016/j.jrp.2006.01.006

Statista. (2020a). Leading countries based on number of Twitter users as of July 2020.

Retrieved from https://www.statista.com/statistics/242606/number-of-active-twitterusers-in-selected-countries/

Statista. (2020b). Twitter: distribution of global audiences 2020, by age group. Retrieved August 5, 2020, from https://www.statista.com/statistics/283119/age-distribution-ofglobal-twitter-users/

Steegen, S., Tuerlinckx, F., Gelman, A., \& Vanpaemel, W. (2016). Increasing Transparency Through a Multiverse Analysis. Perspectives on Psychological Science, 11(5), 702-712. https://doi.org/10.1177/1745691616658637

Sutherland, S. L., \& Cimpian, A. (2015). An explanatory heuristic gives rise to the belief that words are well suited for their referents. Cognition, 143, 228-240. https://doi.org/https://doi.org/10.1016/j.cognition.2015.07.002

Swanson, S. J., Conant, L. L., Humphries, C. J., LeDoux, M., Raghavan, M., Mueller, W. M., ... Binder, J. R. (2020). Changes in description naming for common and proper nouns after left anterior temporal lobectomy. Epilepsy \& Behavior, 106, 106912. https://doi.org/https://doi.org/10.1016/j.yebeh.2020.106912

Sylwester, K., \& Purver, M. (2015). Twitter Language Use Reflects Psychological Differences between Democrats and Republicans. PLOS ONE, 10(9), e0137422. Retrieved from https://doi.org/10.1371/journal.pone.0137422

Terrizzi, J. A., Shook, N. J., \& McDaniel, M. A. (2013). The behavioral immune system and social conservatism: a meta-analysis. Evolution and Human Behavior, 34(2), 99-108. https://doi.org/https://doi.org/10.1016/j.evolhumbehav.2012.10.003

Twitter. (2020). Twitter Privacy Policy.

van Leeuwen, F., \& Park, J. H. (2009). Perceptions of social dangers, moral foundations, and political orientation. Personality and Individual Differences, 47(3), 169-173. https://doi.org/https://doi.org/10.1016/j.paid.2009.02.017

Walton, G. M., \& Banaji, M. R. (2004). Being What You Say: The Effect of Essentialist Linguistic Labels on Preferences. Social Cognition, 22(2), 193-213. https://doi.org/10.1521/soco.22.2.193.35463

Webster, D. M., \& Kruglanski, A. W. (1994). Individual differences in need for cognitive closure. Journal of Personality and Social Psychology, 67(6), 1049-1062.

Webster, D. M., Kruglanski, A. W., \& Pattison, D. A. (1997). Motivated language use in intergroup contexts: Need-for-closure effects on the linguistic intergroup bias. Journal of Personality and Social Psychology. US: American Psychological Association. https://doi.org/10.1037/0022-3514.72.5.1122

Wojcik, S. P., Hovasapian, A., Graham, J., Motyl, M., \& Ditto, P. H. (2015). Conservatives report, but liberals display, greater happiness. Science, 347(6227), 1243 LP - 1246. https://doi.org/10.1126/science.1260817

Wu, S., Hofman, J. M., Mason, W. A., \& Watts, D. J. (2011). Who Says What to Whom on Twitter. In Proceedings of the 20th International Conference on World Wide Web (pp. 705-714). New York, NY, USA: Association for Computing Machinery. https://doi.org/10.1145/1963405.1963504

Zamal, F. Al, Liu, W., \& Ruths, D. (2012). Homophily and Latent Attribute Inference: Inferring Latent Attributes of Twitter Users from Neighbors. ICWSM, 270(2012).

Zmigrod, L., Rentfrow, P. J., \& Robbins, T. W. (2018). Cognitive underpinnings of nationalistic ideology in the context of Brexit. Proceedings of the National Academy of Sciences, 115(19), E4532-E4540. https://doi.org/10.1073/pnas.1708960115 
Zmigrod, L., Rentfrow, P. J., \& Robbins, T. W. (2020). The partisan mind: Is extreme political partisanship related to cognitive inflexibility? Journal of Experimental Psychology: General, 149(3), 407-418. https://doi.org/10.1037/xge0000661 